\title{
Imaging tumour hypoxia with positron emission tomography
}

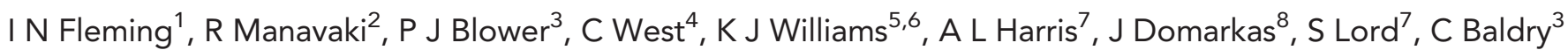
and F J Gilbert ${ }^{\star 2,6}$

${ }^{1}$ Aberdeen Biomedical Imaging Centre, Lilian Sutton Building, Foresterhill, Aberdeen AB25 2ZD, UK; ${ }^{2}$ Department of Radiology, School of Clinical Medicine, University of Cambridge, Box 218-Cambridge Biomedical Campus, Cambridge CB2 0QQ, UK; ${ }^{3}$ Division of Imaging Sciences and Biomedical Engineering, St Thomas' Hospital, King's College London, 4th Floor, Lambeth Wing, London SE1 7EH, UK; ${ }^{4}$ Manchester Academic Health Science Centre, Institute of Cancer Sciences, University of Manchester, Wilmslow Road, Manchester M20 4BX, UK; ${ }^{5}$ Manchester Pharmacy School, Faculty of Medical and Human Sciences, University Manchester, Stopford Building, Oxford Road, Manchester M13 9PT, UK; ${ }^{6}$ EPSRC and CRUK Cancer Imaging Centre in Cambridge and Manchester, Cambridge, UK; ${ }^{7}$ Molecular Oncology Laboratories, University Department of Medical Oncology, The Weatherall Institute of Molecular Medicine, University of Oxford, John Radcliffe Hospital, Headington, Oxford OX3 9DS, UK and ${ }^{8}$ Centre for Cardiovascular and Metabolic Research, Respiratory Medicine, Hull-York Medical School, University of Hull, Hull HU16 5JQ, UK
\end{abstract}

Hypoxia, a hallmark of most solid tumours, is a negative prognostic factor due to its association with an aggressive tumour phenotype and therapeutic resistance. Given its prominent role in oncology, accurate detection of hypoxia is important, as it impacts on prognosis and could influence treatment planning. A variety of approaches have been explored over the years for detecting and monitoring changes in hypoxia in tumours, including biological markers and noninvasive imaging techniques. Positron emission tomography (PET) is the preferred method for imaging tumour hypoxia due to its high specificity and sensitivity to probe physiological processes in vivo, as well as the ability to provide information about intracellular oxygenation levels. This review provides an overview of imaging hypoxia with PET, with an emphasis on the advantages and limitations of the currently available hypoxia radiotracers.

Low oxygen concentration (hypoxia) is associated with many human pathological processes, including ischaemic heart disease, stroke and cancer. In oncology, hypoxic tumours are associated with a poor prognosis, an aggressive phenotype, increased risk of invasion and metastasis, and resistance to chemo and radiation therapy. A practical, robust and reproducible method of detecting and quantifying hypoxia could improve patient outcomes by allowing selection of more appropriate therapies to overcome the effects of hypoxia or allowing stratification of patients for more accurate prognostic information.

Tumour hypoxia has been studied with various techniques: oxygen electrodes; extrinsic (e.g., pimonidazole) and intrinsic (e.g., carbonic anhydrase IX, CAIX) biomarkers; blood oxygen leveldependent (BOLD) and tissue oxygen level-dependent (TOLD) magnetic resonance imaging (MRI); single photon emission computed tomography (SPECT) and positron emission tomography (PET). Each technique interrogates different aspects of the hypoxic microenvironment, as they provide information on hypoxia at different locations: PET, SPECT and extrinsic markers, report on intracellular hypoxia (although not specifically inside cell nuclei and PET/SPECT images quantify data on a macroscopic scale in tumour regions), BOLD-MRI allows assessment of blood oxygenation using deoxy-haemoglobin as an endogenous marker, while oxygen electrodes, OxyLite sampling and electron paramagnetic resonance (EPR) predominantly measure interstitial hypoxia. Indirect methods that report on hypoxia-induced molecular events (e.g., GLUT1, CAIX expression) rather than hypoxia itself have also been employed as markers of tumour oxygenation. Positron emission tomography displays some advantages for studying hypoxia, as it can employ radiotracer probes that 
directly report on oxygen levels, in principle permitting the noninvasive and three-dimensional assessment of intratumour oxygen levels in a more direct manner, and not via hypoxia-mediated changes in phenotype.

Due to the clinical significance of hypoxia imaging, an increasing number of hypoxia PET tracers are being evaluated in the clinic. This review provides a summary and discussion of tumour hypoxia imaging with PET, emphasising the attributes and limitations of the currently available hypoxia radiotracers.

\section{THE SIGNIFICANCE OF TUMOUR HYPOXIA}

Tissue hypoxia is the result of inadequate tissue oxygenation due to an imbalance between oxygen supply and consumption. Hypoxia in solid tumours is largely due to the decreased delivery of oxygenated blood to meet the increased metabolic demands of the rapidly proliferating tumour cells. Other pathogenetic factors preeminent in the aetiology of tumour hypoxia lie in the chaotic and primitive tumour microvasculature, which exhibits severe structural and functional abnormalities, heterogeneous microcirculation patterns, and an adverse geometry that poses limitations to oxygen diffusion. In addition, the reduced oxygen binding ability and/or transport capacity of haemoglobin, due to rouleaux formation, and the presence of disease- or therapy-related anaemia may also exacerbate hypoxia (Vaupel and Harrison, 2004).

Tumour hypoxia may be broadly classified as chronic and acute. Chronic or diffusion-limited hypoxia primarily arises as a consequence of the disorganised vascular architecture of tumours, where the distances between tumour microvessels are often increased from normal. Consequently, the diffusion distances of oxygen in perivascular space-typically $70-180 \mu \mathrm{m}$ from the nearest capillary-are often exceeded. In addition, an adverse vascular geometry and prolonged reductions in blood oxygen content due to anaemia can also result in chronic hypoxia. By contrast, acute or perfusion-limited hypoxia is characterised by fluctuations in tumour blood flow that are caused by transient reductions in perfusion. Both chronic and acute hypoxia can concur in tumours, leading to the formation of a highly dynamic microenvironment, where cells are exposed to differential oxygen gradients both spatially and temporally (Vaupel and Harrison, 2004). Owing to the dynamic and heterogeneous character of tumour hypoxia, imaging with PET presents an attractive alternative, as it does not require invasive biopsies, provides information across the entire tumour, and allows repeated and quantifiable measurements.

Hypoxia has been shown to change gene expression to favour survival in a hostile environment (Bristow and Hill, 2008). The cellular response to hypoxia is mainly controlled by the family of hypoxia-inducible factors (HIFs), and may involve regulation of up to $1.5 \%$ of the human genome. HIF-1-the best characterised member of the HIF family-is a heterodimeric protein, consisting of an oxygen responsive $\alpha$-subunit and a constitutively expressed $\beta$-subunit. In the presence of oxygen, HIF- $1 \alpha$ is continuously synthesised and degraded, but under hypoxic conditions, the protein accumulates, heterodimerises, and acts as a transcription factor to upregulate a multitude of genes, including those involved in glucose metabolism, $\mathrm{pH}$ regulation, apoptosis, cell survival under oxidative stress, angiogenesis, and erythropoiesis (Semenza, 2004). These characteristics eventually confer tumours with resistance to chemoradiation therapy and higher degrees of invasiveness. Furthermore, hypoxia itself reduces free radical formation induced by radiation, providing a physical contribution to resistance. Several retrospective immunohistochemical studies have demonstrated that hypoxia-mediated expression of HIF- $1 \alpha$ and its downstream genes (e.g., glucose transporter 1, GLUT-1; vascular endothelial factor, VEGF; CAIX) is a negative prognostic indicator for many cancer types (Jubb et al, 2010). Treatment resistance to radio and chemotherapy has also been demonstrated. Radiotherapy relies on the formation of free radicals that cause DNA damage; a mechanism that is enhanced in the presence of oxygen. Chemotherapeutic resistance may also be explained by a multitude of mechanisms, including extracellular acidification, resistance to apoptosis, and increased genomic instability. Consequently, patients with hypoxic tumours often have a poor prognosis and decreased overall survival rate.

\section{MEASURING TUMOUR HYPOXIA WITH PET}

Radionuclide detection of hypoxia in tumours was first reported in 1981 with ${ }^{14} \mathrm{C}$-misonidazole autoradiography (Chapman, 1979). Subsequently, two main tracer classes have been developed to specifically study hypoxia with PET: ${ }^{18} \mathrm{~F}$-labelled nitroimidazoles and $\mathrm{Cu}$-labelled diacetyl-bis $\left(N^{4}\right.$-methylthiosemicarbazone) analogues (Figure 1).

From a PET imaging perspective, hypoxia markers need to exhibit a number of different properties. The tracer must readily and non-specifically enter cells, sample the intracellular milieu, and leave cells only in the presence of relevant oxygen concentrations. A summary of the attributes of the ideal hypoxia tracer is presented in Table 1. Most PET tracers tested clinically broadly display attributes $1,4,5$, and 7 . The clinical utility of each tracer depends on these key properties, which will influence its distribution in tissues, clearance rate from blood, normoxic and hypoxic cells, metabolism, optimal image acquisition time and ease of synthesis, distribution.

\section{NITROIMIDAZOLE ANALOGUES}

2-Nitroimidazole compounds were originally developed as hypoxic cell radiosensitisers and were introduced as hypoxia markers in the 1970s (Chapman, 1979). Nitroimidazoles enter cells by passive diffusion, where they undergo reduction forming a reactive intermediate species. Under normoxic conditions, these molecules are re-oxidised into their parent compound and diffuse out of the cell. However, hypoxia causes further reduction of the nitro-radical anion, which eventually becomes irreversibly trapped in the cell at rates that are inversely proportional to the local $\mathrm{pO}_{2}$. As reduction of nitroimidazoles requires the presence of active tissue reductases, these compounds accumulate within viable hypoxic cells, but not apoptotic or necrotic cells.

${ }^{18}$ F-fluoromisonidazole. Over the years, several fluorinated nitroimidazole-based markers have been developed for PET imaging. Of these, ${ }^{18} \mathrm{~F}$-fluoromisonidazole $\left({ }^{18} \mathrm{~F}\right.$-FMISO) constitutes the prototype 2-nitroimidazole tracer, and is the most extensively clinically studied PET hypoxia biomarker. The lipophilic nature of this compound ensures facile cell-membrane penetration and diffusion into tissue, and several studies correlating direct oxygen measurements with ${ }^{18} \mathrm{~F}$-FMISO accumulation in vivo demonstrate that a median oxygen level of $\leqslant 10 \mathrm{~mm} \mathrm{Hg}$ is generally required for hypoxia-specific retention. The ${ }^{18} \mathrm{~F}$-FMISO accumulation has been found to reflect hypoxia in gliomas (Valk et al, 1992; Bruehlmeier et al, 2004; Rajendran et al, 2004; Cher et al, 2006; Swanson et al, 2009), head-and-neck (Rasey et al, 1996; Gagel et al, 2004, 2007; Hicks et al, 2005; Thorwarth et al, 2006; Zimny et al, 2006; Mortensen et al, 2010; Abolmaali et al, 2011; Sato et al, 2013), breast (Cheng et al, 2013), lung (Cherk et al, 2006; Vera et al, 2011), and renal tumours (Hugonet et al, 2011). However, ${ }^{18} \mathrm{~F}$ FMISO retention in sarcomas is variable (Rajendran et al, 2003; Mortensen et al, 2010), rectal ${ }^{18} \mathrm{~F}$-FMISO imaging is compromised 
<smiles>O=[N+]([O-])c1nccn1CC(O)CF</smiles>

FMISO (logP:0.4)<smiles>O=[N+]([O-])c1nccn1C1OC(CF)C(O)C1O</smiles>

FAZA (logP:-0.4)<smiles>O=[N+]([O-])c1nccn1CC(O)C(O)CF</smiles>

FETNIM (logP:-0.77)<smiles>O=C(Cn1ccnc1[N+](=O)[O-])NCCC(F)(F)F</smiles>

EF3 (logP:0.05)<smiles>O=C(Cn1ccnc1[N+](=O)[O-])NCC(F)(F)F</smiles>

EF5 ( $\log \mathrm{P}: 0.6)$<smiles>O=[N+]([O-])c1nccn1COC(CO)CF</smiles>

FRP-170 (logP:0.094)<smiles>O=[N+]([O-])c1nccn1Cc1cn(C(CO)CF)nn1</smiles>

HX4 (logP:-0.69)<smiles></smiles>

Cu-ATSM (logP:1.48)

Figure 1. Structures and $\log P$-values of PET hypoxia radiotracers. The $\log P$-value (partition coefficient) of each radiotracer is shown in the parentheses. Positive log $P$-values indicate a lipophilic molecule, whereas negative log $P$-values represent a hydrophilic molecule.

\begin{tabular}{|l|l|}
\hline \multicolumn{2}{|l|}{ Table 1. Characteristics of the ideal hypoxia tracer } \\
\hline 1 & Hypoxia-specific retained in regions with low $\mathrm{pO}_{2}$ levels, but not by normoxic or necrotic cells \\
\hline 2 & Mechanism of cellular retention should be well defined and cell type independent \\
\hline 3 & $\begin{array}{l}\text { Sufficiently lipophilic to enter cells and allow uniform tissue distribution, but also sufficiently hydrophilic to avoid membrane sequestration, and have faster } \\
\text { clearance from systemic circulation and normoxic tissue }\end{array}$ \\
\hline 4 & Pharmacokinetic profile and tissue distribution should exhibit little dependence on parameters that may co-vary with hypoxia, such as blood flow or pH \\
\hline 5 & High stability against non-hypoxia specific metabolism in vivo \\
\hline 6 & Tissue kinetics should be suitable to imaging within a timeframe permitted in the clinical setting \\
\hline 7 & Should be easy to synthesise and readily available \\
\hline 8 & Amenable dosimetry profile \\
\hline 9 & Be repeatable to allow both detection of hypoxia and return to normoxia \\
\hline 10 & Should be effective in multiple tumour types \\
\hline $\mathrm{pO}_{2}:$ partial oxygen pressure (mm Hg). \\
\hline
\end{tabular}

by high non-specific tracer accumulation in normoxic tissue (Roels et al, 2008) whereas no retention was observed in pancreatic tumours (Segard et al, 2013). Several clinical studies have shown that a tumour-to-blood activity ratio of $\geqslant 1.2$ imaged after at least $2 \mathrm{~h}$ post injection (p.i.) can be generally considered as indicative of hypoxia (Table 2). Although not commercially available, ${ }^{18} \mathrm{~F}$ FMISO is produced by a number of institutions, making it available for research purposes.

Due to its hypoxic selectivity, ${ }^{18}$ F-FMISO is the lead candidate in the assessment of hypoxia with PET. However, despite its wide applicability, ${ }^{18}$ F-FMISO has not gained general acceptance for routine clinical use due to its slow pharmacokinetic profile: the limited clearance of the tracer from normal tissue and blood results in modest hypoxic-to-normoxic tissue ratios (Figure 2) and therefore images with moderate contrast (Figure 3A). The limited hypoxic contrast may potentially impede visual detection of hypoxic regions, and has hampered diagnostic utility in routine practice. Therefore, considerable efforts have been made to develop hypoxia markers with improved pharmacokinetic properties (enhanced clearance of the tracer from normoxic tissues) that are more amenable to clinical use. These are discussed below.

${ }^{18}$ F-fluoroazomycin-arabinofuranoside. ${ }^{18} \mathrm{~F}$-fluoroazomycin-arabinofuranoside $\left({ }^{18} \mathrm{~F}\right.$-FAZA) is more hydrophilic than ${ }^{18} \mathrm{~F}$-FMISO.
Consequently, there are faster clearance kinetics, resulting in improved tumour-to-reference tissue ratios, and thus hypoxia-to-normoxia contrast. The ${ }^{18}$ F-FAZA imaging has been successful in gliomas (Postema et al, 2009), lymphomas (Postema et al, 2009), lung (Postema et al, 2009; Bollineni et al, 2013; Trinkaus et al, 2013), head-and-neck (Grosu et al, 2007; Souvatzoglou et al, 2007; Postema et al, 2009; Mortensen et al 2012), cervical (Schuetz et al, 2010), and rectal tumours (Havelund et al, 2013), and results have been shown to compare favourably with equivalent ${ }^{18}$ F-FMISO data, especially as improved hypoxic-normoxic contrast was obtained at earlier time points. No ${ }^{18}$ F-FAZA accumulation has been observed in prostate tumours, although hypoxia may not be a characteristic of this particular tumour type, as in the same study, CAIX immunohistochemistry was also found to be negative in these lesions (Garcia-Parra et al, 2011). High ${ }^{18}$ F-FAZA tumour-toreference tissue values have been associated with reduced disease-free survival and have shown prognostic potential in the detection of hypoxia in head-and-neck patients (Mortensen et al, 2012). Due to the higher tumour-to-reference tissue ratios in comparison with ${ }^{18} \mathrm{~F}$-FMISO, ${ }^{18} \mathrm{~F}$-FAZA is gaining popularity for PET imaging of tumour hypoxia. Despite the fact that ${ }^{18} \mathrm{~F}$ FAZA is not widely available at present, increasing research demand may persuade more sites to produce it. 
Table 2. Clinical hypoxia studies with PET in tumours

\begin{tabular}{|c|c|c|c|c|c|}
\hline Reference & Tracer & $\begin{array}{l}\text { Tumour } \\
\text { type(s) }\end{array}$ & $N$ & Tracer retention (TBR; SUV) & Results \\
\hline Valk et al (1992) & ${ }^{18} \mathrm{~F}-\mathrm{FMISO}$ & Brain & 3 & $\mathrm{~T}: \mathrm{P}: 0.71-1.49$ at $120 \mathrm{~min}$ p.i. & $\begin{array}{l}{ }^{18} \mathrm{~F} \text {-FMISO-PET is a feasible method for detecting } \\
\text { hypoxia in gliomas }\end{array}$ \\
\hline Bruehlmyer et al (2004) & ${ }^{18} \mathrm{~F}-\mathrm{FMISO}$ & Brain & 11 & $\begin{array}{l}\mathrm{T}: \mathrm{B}: 0.96-2.07 \text { at } 90 \mathrm{~min} \text { and } \\
\geqslant 170 \mathrm{~min} \text { p.i. }\end{array}$ & $\begin{array}{l}\text { Increased }{ }^{18} \mathrm{~F} \text {-FMISO T:B observed in all tumours. T:B } \\
\text { independent of tumour perfusion at later imaging times }\end{array}$ \\
\hline Cher et al (2006) & ${ }^{18} \mathrm{~F}-\mathrm{FMISO}$ & Brain & 17 & Static scan at 120 min p.i. & $\begin{array}{l}{ }^{18} \mathrm{~F} \text {-FMISO uptake in high-grade, but not in low-grade, } \\
\text { gliomas. Correlation between }{ }^{18} \mathrm{~F}-\mathrm{FDG} \text { or }{ }^{18} \mathrm{~F}-\mathrm{FMISO} \\
\text { uptake with Ki67 and VEGFR-1 expression }\end{array}$ \\
\hline Swanson et al (2009) & ${ }^{18} \mathrm{~F}-\mathrm{FMISO}$ & Brain & 24 & $\begin{array}{l}T: B_{\max , \text { pre-therapy }}: 2.7 \\
T: B_{\max , \text { post-therapy }}: 1.7\end{array}$ & $\begin{array}{l}\text { Hypoxia volume generally straddled outer edge of the } \\
\text { T1-Gd abnormality. Correlation between hypoxic } \\
\text { volume and T1-Gd abnormality. }{ }^{18} \mathrm{~F}-\mathrm{FMISO} \text { T:B reduced } \\
\text { after therapy }\end{array}$ \\
\hline Cheng et al (2013) & ${ }^{18} \mathrm{~F}-\mathrm{FMISO}$ & Breast & 20 & 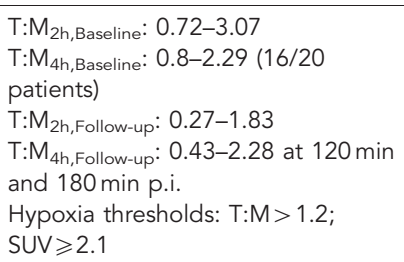 & $\begin{array}{l}\text { Correlation between FMISO uptake and endocrine } \\
\text { therapy outcome. Poor correlation between FMISO } \\
\text { uptake and HIF-1a immunostaining }\end{array}$ \\
\hline Gagel et al (2004) & ${ }^{18} \mathrm{~F}-\mathrm{FMISO}$ & $\mathrm{H} \& N$ & 16 & $\begin{array}{l}\text { T:M: } 1.68 \text { (range, } 1.23-2.28 \text { ) } \\
\text { Av SUV } \text { mean: } 1.76 ; A v . S U V_{\text {max }}: 2.07 \\
\text { at } 120 \text { min p.i. }\end{array}$ & $\begin{array}{l}\text { Average to high correlation between oxygen electrode } \\
\text { and }{ }^{18} \mathrm{~F}-\mathrm{FMISO} \mathrm{T} \text { :M and SUV. No correlation between } \\
\text { tumour oxygenation status and }{ }^{18} \mathrm{~F} \text {-FDG uptake }\end{array}$ \\
\hline Hicks et al (2005) & ${ }^{18} \mathrm{~F}-\mathrm{FMISO}$ & $\mathrm{H} \& \mathrm{~N}$ & 15 & $\begin{array}{l}\text { SUV }_{\max } \\
\text { Tumour: } 2.5 \pm 0.5 \\
\text { Nodes: } 2.3 \pm 0.5 \text { at } 120 \mathrm{~min} \text { p.i. }\end{array}$ & $\begin{array}{l}\text { Positive }{ }^{18} \mathrm{~F} \text {-FMISO uptake in } 13 \text { patients. Qualitative } \\
\text { decrease in }{ }^{18} \mathrm{~F} \text {-FMISO and }{ }^{18} \mathrm{~F} \text {-FDG uptake induced by } \\
\text { therapy }\end{array}$ \\
\hline Thorwarth et al (2005) & ${ }^{18} \mathrm{~F}-\mathrm{FMISO}$ & $H \& N$ & 15 & $\begin{array}{l}\text { Median SUV } V_{\text {max }}: 2.25 \text { (range, } 1.36- \\
4.04 \text { ) at } 120 \text { min and } 180 \text { min p.i. }\end{array}$ & $\begin{array}{l}\text { Different types of characteristic hypoxia-perfusion } \\
\text { patterns identified in tumours }\end{array}$ \\
\hline Rajendran et al (2006) & ${ }^{18} \mathrm{~F}-\mathrm{FMISO}$ & $\mathrm{H} \& \mathrm{~N}$ & 73 & Mean $\mathrm{T}: \mathrm{B}_{\max } 1.6 \pm 0.46$ & $\begin{array}{l}\mathrm{T}: \mathrm{B} \text { and the presence of nodes were strong independent } \\
\text { predictors of survival }\end{array}$ \\
\hline Rischin et al (2006) & ${ }^{18} \mathrm{~F}-\mathrm{FMISO}$ & $\mathrm{H} \& N$ & 45 & $\begin{array}{l}\text { Independent hypoxic score } \\
\text { Static scan at } 120 \text { min p.i. }\end{array}$ & $\begin{array}{l}\text { Higher risk of locoregional failure in hypoxic tumours. } \\
\text { Patients on tirapazamine had lower risk of locoregional } \\
\text { failure }\end{array}$ \\
\hline Thorwarth et al (2006) & ${ }^{18} \mathrm{~F}-\mathrm{FMISO}$ & $\mathrm{H} \& N$ & 12 & $\begin{array}{l}\text { SUV }_{\text {max }}: 2.20 \text { (range, } 1.4-3.22 \text { ) at } \\
120 \text { min and } 240 \text { min p.i } \\
\text { Hypoxia definition: } S U V>1.4\end{array}$ & $\begin{array}{l}\text { No correlation between }{ }^{18} \mathrm{~F}-\mathrm{FDG} \text { and }{ }^{18} \mathrm{~F}-\mathrm{FMISO} \text { SUV. } \\
\text { Maximum }{ }^{18} \mathrm{~F} \text {-FMISO SUV showed borderline } \\
\text { significance for stratifying patient group }\end{array}$ \\
\hline Zimny et al (2006) & ${ }^{18} \mathrm{~F}-\mathrm{FMISO}$ & $\mathrm{H} \& \mathrm{~N}$ & 24 & $\begin{array}{l}\text { Normoxic } T: M_{\text {mean }} 1.4 \\
\text { Hypoxic } T: M_{\text {mean }}: 1.8\end{array}$ & $\begin{array}{l}{ }^{18} \mathrm{~F} \text {-FMISO T:M higher in hypoxic tumours (as detected } \\
\text { with oxygen electrode). Moderate correlation between } \\
{ }^{18} \mathrm{~F} \text {-FDG and }{ }^{18} \mathrm{~F} \text {-FMISO uptake }\end{array}$ \\
\hline Eschmann et al (2007) & ${ }^{18} \mathrm{~F}-\mathrm{FMISO}$ & $H \& N$ & 14 & 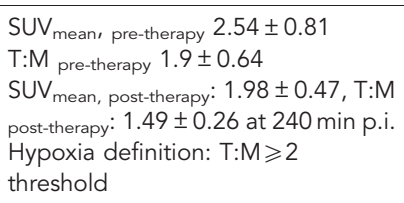 & Radiotherapy decreased ${ }^{18} \mathrm{~F}-\mathrm{FMISO}$ SUV and T:M ratio \\
\hline Gagel et al (2007) & ${ }^{18} \mathrm{~F}-\mathrm{FMISO}$ & $\mathrm{H} \& \mathrm{~N}$ & 38 & $\begin{array}{l}S U V_{\text {mean }}: 1.69 \\
S U V_{\text {max }}: 1.98 \\
T: M_{\text {mean }}: 1.57 \\
T: B_{\text {mean }}: 1.13\end{array}$ & $\begin{array}{l}\text { Moderate correlation between oxygen measurements } \\
\text { and }{ }^{18} \mathrm{~F} \text {-FMISO uptake. Low correlation between } \\
{ }^{18} \mathrm{~F} \text {-FDG and }{ }^{18} \mathrm{~F} \text {-FMISO }\end{array}$ \\
\hline Lee et al (2008) & ${ }^{18} \mathrm{~F}-\mathrm{FMISO}$ & $\mathrm{H} \& N$ & 20 & $\begin{array}{l}\text { Static scan at } 120-150 \text { min p.i. } \\
\text { Hypoxia definition: } T: M \geqslant 1.3\end{array}$ & Variable ${ }^{18} \mathrm{~F}$-FMISO distribution \\
\hline Nehmeh et al (2008) & ${ }^{18} \mathrm{~F}-\mathrm{FMISO}$ & $\mathrm{H} \& N$ & 13 & $\begin{array}{l}\text { SUV } 1.9-4.5 \text { at } 117-195 \text { p.i. } \\
T B R \geqslant 1.2\end{array}$ & $\begin{array}{l}\text { Good correlations intratumour }{ }^{18} \mathrm{~F} \text {-FMISO distributions in } \\
6 / 13 \text { patients, consistent with chronic hypoxia }\end{array}$ \\
\hline Dirix et al (2009) & ${ }^{18} \mathrm{~F}-\mathrm{FMISO}$ & $H \& N$ & 15 & $\begin{array}{l}\text { Hypoxic volume } \\
\text { T: } B_{\max } \text { pre-therapy } 4.1 \mathrm{ml} \text {, } \\
\text { Hypoxic volume } 1.5 \\
\mathrm{~T}: \mathrm{B}_{\max , \text { post-therapy }}: 1.2 \text { at } 120- \\
160 \text { min p.i } \\
\text { Hypoxia definition: } \mathrm{T}: \mathrm{B}>1.2\end{array}$ & $\begin{array}{l}\text { Disease-free survival correlates negatively with baseline } \\
T: B_{\max } \text { and initial hypoxic volume }\end{array}$ \\
\hline Lee et al (2009) & ${ }^{18} \mathrm{~F}-\mathrm{FMISO}$ & $H \& N$ & 28 & - & $\begin{array}{l}\text { Heterogeneous distribution of }{ }^{18} \mathrm{~F}-\mathrm{FMISO} \text { noted in the } \\
\text { primary and/or nodal disease in } 90 \% \text { of patients }\end{array}$ \\
\hline Abolmaali et al (2011) & ${ }^{18} \mathrm{~F}-\mathrm{FMISO}$ & $\mathrm{H} \& \mathrm{~N}$ & 23 & 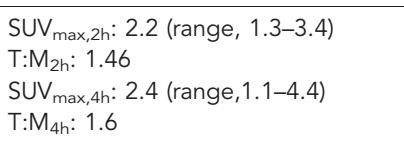 & ${ }^{18} \mathrm{~F}-\mathrm{FMISO}$ contrast increases $2-4 \mathrm{~h}$ p.i. \\
\hline
\end{tabular}


Table 2. (Continued)

\begin{tabular}{|c|c|c|c|c|c|}
\hline Reference & Tracer & $\begin{array}{l}\text { Tumour } \\
\text { type(s) }\end{array}$ & $N$ & Tracer retention (TBR; SUV) & Results \\
\hline Kikuchi et al (2011) & ${ }^{18} \mathrm{~F}-\mathrm{FMISO}$ & H\&N & 17 & $\begin{array}{l}\text { Median SUV } \max : 2.3 \\
\text { Median T:M: } 1.3 \text { at } 150 \text { min p.i. } \\
\text { Hypoxia definition: } 1.3\end{array}$ & $\begin{array}{l}\text { Disease-specific survival was significantly lower in } \\
\text { patient group with high basal }{ }^{18} \mathrm{~F} \text {-FMISO SUV } \mathrm{max}_{\max } \text { and } \\
\mathrm{T}: \mathrm{M}_{\max }\end{array}$ \\
\hline Yamane et al (2011) & ${ }^{18} \mathrm{~F}-\mathrm{FMISO}$ & $\mathrm{H} \& \mathrm{~N}$ & 13 & $\begin{array}{l}\text { SUV }_{\text {max,pre-therapy }} 2.2 \text { (range, } \\
0.7-3.6 \text { ) } \\
\text { T:M,pre-therapy: } 1.6 \text { (range: } 1.1-2.2 \text { ). } \\
\text { Responders: }-18.7 \% \mathrm{SUV}_{\text {maxi }} \\
-22.5 \% \mathrm{~T}: \mathrm{M} ;-82.65 \% \text { hypoxic } \\
\text { volume non-responders: }-5.5 \% \text { SUV }_{\max } \\
10.2 \% \mathrm{~T}: \mathrm{M} \\
-8.8 \% \text { hypoxic volume }(-/+ \text { denote } \\
\% \text { increase and decrease, } \\
\text { respectively) at } 150 \text { min p.i. }\end{array}$ & $\begin{array}{l}{ }^{18} \mathrm{~F} \text {-FMISO SUV } \\
\text { significantly decreased after neo-adjuvant } \\
\text { chemotherapy }\end{array}$ \\
\hline Sato et al (2013) & ${ }^{18} \mathrm{~F}-\mathrm{FMISO}$ & $\mathrm{H} \& \mathrm{~N}$ & 23 & $\begin{array}{l}\text { Median SUV } V_{\text {max: }} 1.83 \text { (range, } \\
0.8-2.7 \text { ) } \\
\text { Median SUV } \\
1.0-32.3 \text { max: } 16.5 \text { (range, }\end{array}$ & $\begin{array}{l}\text { Weak significant correlation between }{ }^{18} \mathrm{~F} \text {-FMISO and } \\
{ }^{18} \mathrm{~F} \text {-FDG SUV }{ }_{\text {max. }}{ }^{18} \mathrm{~F} \text {-FMISO SUV } \max \text { was significantly } \\
\text { higher in HIF- } \alpha \text {-positive cases than in HIF- } 1 \alpha \text {-negative } \\
\text { cases }\end{array}$ \\
\hline Okamoto et al (2013) & ${ }^{18} \mathrm{~F}-\mathrm{FMISO}$ & $\mathrm{H} \& \mathrm{~N}$ & 11 & 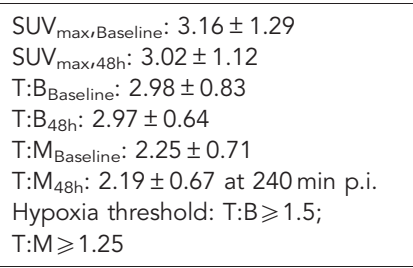 & $\begin{array}{l}\text { High reproducibility between SUV, T:B, T:M and hypoxic } \\
\text { volume measurements between the two }{ }^{18} \mathrm{~F}-\mathrm{FMISO} \\
\text { scans (baseline and at } 48 \mathrm{~h} \text { ) }\end{array}$ \\
\hline Mortensen et al (2010) & ${ }^{18} \mathrm{~F}-\mathrm{FMISO}$ & $\begin{array}{l}\text { H\&N } \\
\text { Sarcoma }\end{array}$ & 19 & $\begin{array}{l}\mathrm{T}: \mathrm{M}_{\text {med: }}: \mathrm{H} \& \mathrm{~N}: 1.68 \text { (range, } \\
0.7-2.38 \text { ) } \\
\text { Sarcoma: } 0.78 \text { (range, } 0.7-1 \text { ) }\end{array}$ & $\begin{array}{l}\text { No correlation between }{ }^{18} \text { F-FMISO retention and } \\
\text { oxygen electrode }\end{array}$ \\
\hline Koh et al (1995) & ${ }^{18} \mathrm{~F}$-FMISO & Lung & 7 & $\begin{array}{l}\text { Static scan at } 120-180 \text { p.i. } \\
\text { TBR } \geqslant 1.4 \text { threshold to define } \\
\text { hypoxia }\end{array}$ & $\begin{array}{l}\text { Radiotherapy reduced median fractional hypoxic volume } \\
\text { from } 58 \text { to } 22 \%\end{array}$ \\
\hline Cherk et al (2006) & ${ }^{18} \mathrm{~F}-\mathrm{FMISO}$ & Lung & 21 & $\begin{array}{l}\text { SUV: } 0.4-2.14 ; \text { T:N: } 1.18-9.73 \text { at } \\
120 \text { min p.i. }\end{array}$ & $\begin{array}{l}\text { Low }{ }^{18} \mathrm{~F} \text {-FMISO uptake. Poor correlation between } \\
{ }^{18} \mathrm{~F} \text {-FMISO and }{ }^{18} \mathrm{~F} \text {-FDG uptake }\end{array}$ \\
\hline Gagel et al (2006) & ${ }^{18} \mathrm{~F}$-FMISO & Lung & 8 & $\begin{array}{l}\text { SUV }_{\text {mean, pre-therapy: }} 2.31 \pm 0.2 \\
\text { SUV } \\
\text { T:Max, pre-therapy: } 2.77 \pm 0.27 \\
\text { SUV } \\
\text { Sue-therapy: } 1.99 \pm 0.49 \\
\text { SUV } \\
\text { T:Max, post-therapy: } 1.83 \pm 0.12 \\
180 \text { min p.i. }\end{array}$ & $\begin{array}{l}{ }^{18} \mathrm{~F}-\mathrm{FMISO} \text { can define hypoxic sub-regions. Changes in } \\
\text { FMISO and }{ }^{1{ }^{1}} \mathrm{~F} \text {-FDG PET measure early response to } \\
\text { therapy }\end{array}$ \\
\hline Vera et al (2011) & ${ }^{18} \mathrm{~F}-\mathrm{FMISO}$ & Lung & 5 & $\begin{array}{l}\text { SUV }_{\max , \text { pre-therapy }}: 1-2.5 \\
\text { SUV }_{\max , \text { post-therapy }}: 1-2.4\end{array}$ & $\begin{array}{l}{ }^{18} \mathrm{~F} \text {-FMISO uptake higher in tumours than in nodes and } \\
\text { did not change during therapy }\end{array}$ \\
\hline Thureau et al (2013) & ${ }^{18} \mathrm{~F}$-FMISO & Lung & 10 & - & $\begin{array}{l}\text { Low reproducibility and inter-observer agreement for } \\
{ }^{18} \mathrm{~F} \text {-FMISO volume measurements on the basis of visual } \\
\text { scoring. } \mathrm{T}: \mathrm{M} \geqslant 1.4 \text { recommended for hypoxic volume } \\
\text { delineation }\end{array}$ \\
\hline Segard et al (2013) & ${ }^{18} \mathrm{~F}$-FMISO & Pancreatic & 10 & Mean SUV $V_{\max }: 2.3$ (range, 1-3.4) & $\begin{array}{l}{ }^{18} \mathrm{~F} \text {-FMISO accumulation observed in } 2 / 10 \text { patients on } \\
\text { the basis of visual analysis. Minimal }{ }^{18} \mathrm{~F} \text {-FMISO } \\
\text { accumulation in pancreatic tumours; correlation with } \\
\text { other imaging modalities required to allow tumour } \\
\text { localisation and semi-quantitative analysis }\end{array}$ \\
\hline Hugonet et al (2011) & ${ }^{18} \mathrm{~F}$-FMISO & Renal & 53 & $\begin{array}{l}\text { Static scan at } 120 \min \text { p.i. } \\
\text { Hypoxia definition: TBR }>1.2\end{array}$ & Reduction in hypoxic volume post-therapy \\
\hline Roels et al (2008) & ${ }^{18} \mathrm{~F}-\mathrm{FMISO}$ & Rectal & 15 & & $\begin{array}{l}\text { Mismatch between }{ }^{18} \mathrm{~F}-\mathrm{FDG} \text { and }{ }^{18} \mathrm{~F}-\mathrm{FMISO} \text { scans. }{ }^{18} \mathrm{~F}- \\
\text { FMISO uptake reduced after therapy }\end{array}$ \\
\hline Bentzen et al (2003) & ${ }^{18} \mathrm{~F}$-FMISO & Sarcoma & 13 & $\mathrm{~T}: \mathrm{M}<1-1.6$ & $\begin{array}{l}{ }^{18} \mathrm{~F} \text {-FMISO accumulation observed in } 2 / 7 \text { malignant } \\
\text { tumours. } \\
\text { No correlation between }{ }^{18} \mathrm{~F} \text {-FMISO and } \mathrm{pO}_{2} \\
\text { measurements }\end{array}$ \\
\hline Rajendran et al (2003) & ${ }^{18} \mathrm{~F}$-FMISO & Sarcoma & 19 & $\begin{array}{l}\mathrm{T}: \mathrm{B}_{\max } 1.10-3.46 \text { at } 120 \mathrm{~min} \text { p.i. } \\
\mathrm{TBR} \geqslant 1.2 \text { to define hypoxia }\end{array}$ & $\begin{array}{l}{ }^{18} \text { F-FMISO uptake observed in } 14 \text { patients. Poor } \\
\text { correlation between tumour grade, hypoxia volume and } \\
18 \text { F-FDG T:B }\end{array}$ \\
\hline
\end{tabular}




\section{Table 2. (Continued)}

\begin{tabular}{|c|c|c|c|c|c|}
\hline Reference & Tracer & $\begin{array}{l}\text { Tumour } \\
\text { type(s) }\end{array}$ & $N$ & Tracer retention (TBR; SUV) & Results \\
\hline Rajendran et al (2004) & ${ }^{18} \mathrm{~F}-\mathrm{FMISO}$ & $\begin{array}{l}\text { Brain } \\
\text { Breast } \\
\text { H\&N } \\
\text { Sarcoma }\end{array}$ & 49 & $\begin{array}{l}\mathrm{T}: \mathrm{B}_{\max }: \text { Brain } 2.43 \text { (range, } 1.7-2.9 \text { ) } \\
\text { Breast } 1.52 \text { (range, } 0.93-2.6 \text { ) } \\
\mathrm{H} \& N: 1.5 \text { (range, } 0.88-2.4 \text { ) } \\
\text { Sarcoma: } 1.46 \text { (range, } 1.1-2.1 \text { ) }\end{array}$ & $\begin{array}{l}\text { Hypoxia detected in all tumour types. Low correlation } \\
\text { between glucose metabolism and hypoxia }\end{array}$ \\
\hline Schuetz et al (2010) & ${ }^{18}$ F-FAZA & Cervical & 15 & $\begin{array}{l}\mathrm{T}: \mathrm{M}_{\max }: 1.2-3.6 \text { at } 60 \mathrm{~min} \text { and } \\
120 \min \text { p.i. }\end{array}$ & $5 / 15$ patients had visually identifiable tumours. \\
\hline Grosu et al (2007) & ${ }^{18} \mathrm{~F}-\mathrm{FAZA}$ & $H \& N$ & 18 & $\begin{array}{l}\mathrm{T}: \mathrm{M}_{\text {mean }}: 1.6 \\
\mathrm{~T}: \mathrm{M}_{\text {max }}: 2 \text { at } 120 \text { min p.i. } \\
\text { Hypoxia threshold: } \mathrm{SUV} \geqslant 1.5\end{array}$ & $\begin{array}{l}{ }^{18} \mathrm{~F}-\mathrm{FAZA} \text { uptake located in single confluent region in } \\
11 / 18 \text { patients and as multiple diffuse regions in } 4 / 18 \\
\text { patients }\end{array}$ \\
\hline $\begin{array}{l}\text { Souvatzoglou et al } \\
\text { (2007) }\end{array}$ & ${ }^{18} \mathrm{~F}-\mathrm{FAZA}$ & $\mathrm{H} \& \mathrm{~N}$ & 11 & $\begin{array}{l}\left.\text { SUV }_{\text {max }}: 2.3 \text { (range, } 1.5-3.4\right) \\
\text { SUV } \\
\text { T:M: } 2 \text { (range, } 1.6-2.4 \text { (range, } 1-2.1 \text { ) }\end{array}$ & $\begin{array}{l}\text { T:M ratio increased } 60 \text { min p.i. All tumours had } \\
\text { T:M }>1.5 \text {. Tumour volume with } T: M>1.5 \text { was highly } \\
\text { variable }\end{array}$ \\
\hline Mortensen et al (2012) & ${ }^{18}$ F-FAZA & $H \& N$ & 40 & $\begin{array}{l}\text { Median } \mathrm{T}: \mathrm{M}_{\max } 1.5 \text { at } 120 \min \text { p.i. } \\
\text { Hypoxia threshold: } \geqslant 1.4\end{array}$ & $\begin{array}{l}\text { High uptake associated with lower disease-free survival. } \\
\text { Radiotherapy treatment reduced hypoxic volume }\end{array}$ \\
\hline Bollineni et al (2013) & ${ }^{18} \mathrm{~F}-\mathrm{FAZA}$ & Lung & 11 & $\begin{array}{l}\text { Median } T: B: 2.8 \text { (range, } 1.8-4.6 \text { ) } \\
T: B \geqslant 1.2 \text { for hypoxic volume } \\
\text { definition }\end{array}$ & $\begin{array}{l}\text { Not significant correlation between }{ }^{18} \mathrm{~F}-\mathrm{FAZA} \mathrm{T}: \mathrm{B} \text { and } \\
{ }^{18} \mathrm{~F}-\mathrm{FDG} \text { SUV } \\
\text { inax or lesion size. Heterogeneous } \\
\text { intratumoural distribution for }{ }^{18} \mathrm{~F}-\mathrm{FAZA} \text {-based visual } \\
\text { analysis. }{ }^{18} \mathrm{~F}-\mathrm{FAZA} \text { PET is able to detect heterogeneous } \\
\text { distributions of hypoxic sub-volumes }\end{array}$ \\
\hline Trinkhaus et al (2013) & ${ }^{18} \mathrm{~F}-\mathrm{FAZA}$ & Lung & 17 & - & $\begin{array}{l}\text { 11/17 patients had baseline hypoxia based on } \\
\text { qualitative assessment. } 6 / 8 \text { patients with scans following } \\
\text { chemoradiation had resolution of hypoxia on the basis of } \\
\text { qualitative analysis }\end{array}$ \\
\hline $\begin{array}{l}\text { Garcia-Parra et al } \\
\text { (2011) }\end{array}$ & ${ }^{18}$ F-FAZA & Prostate & 14 & $\mathrm{~T}: \mathrm{N}_{\text {mean }}: 1.21$ & $\begin{array}{l}{ }^{18} \text { F-FAZA uptake not increased in tumours. No evidence } \\
\text { of hypoxia as assessed by CaIX IHC staining }\end{array}$ \\
\hline Havelund et al (2013) & ${ }^{18}$ F-FAZA & Rectal & 14 & $\mathrm{~T}: \mathrm{M}_{\text {mean }}: 2.83$ & $\begin{array}{l}{ }^{18} \text { F-FAZA-PET is feasible for visualisation of hypoxia in } \\
\text { rectal cancer }\end{array}$ \\
\hline Postema et al (2009) & ${ }^{18}$ F-FAZA & $\begin{array}{l}\text { H\&N } \\
\text { Lung } \\
\text { Lymphoma } \\
\text { Glioma }\end{array}$ & 50 & $\begin{array}{l}\text { H\&N TBR: } 1.2-2.7 \\
\text { SUV } \\
\text { Lung }_{\max } 1.05-2.35 \\
\text { SUV }_{\max } 0.81-1.93 \\
{\text { Lymphoma TBR: } 1.2-3 ; \text { SUV }_{\max } 1.07-} \text { - } \\
4.52 \\
\text { Glioma TBR: } 1.9-15.6 \mathrm{At} \\
\text { 120-180 min p.i. }\end{array}$ & $\begin{array}{l}\text { High TBR in all } 7 \text { gliomas; high TBR, SUV } \max \text { observed in } \\
6 / 9 \text { H\&N tumours; moderate TBR, SUV } V_{\max } \text { in } 3 / 21 \\
\text { lymphomas; increased TBR, SUV } V_{\max } \text { in } 7 / 11 \text { lung patients }\end{array}$ \\
\hline Lehtiö et al (2001) & ${ }^{18}$ F-FETNIM & $H \& N$ & 8 & $\mathrm{~T}: \mathrm{M}_{\max } 1-4$ at $3 \mathrm{~h}$ p.i. & $\begin{array}{l}\text { Tumour distribution volume correlated strongly with }{ }^{18} \mathrm{~F} \text { - } \\
\text { FETNIM SUV between } 60 \text { and } 120 \text { min p.i. and blood } \\
\text { flow, but not with }{ }^{18} \text { F-FDG SUV. Values compare } \\
\text { favourably with }{ }^{18} \text { F-FMISO data. Late time-point }{ }^{18} \mathrm{~F} \text { - } \\
\text { FETNIM T:M are indicative of hypoxia }\end{array}$ \\
\hline Lehtiö et al (2003) & ${ }^{18}$ F-FETNIM & $\mathrm{H} \& \mathrm{~N}$ & 10 & $\begin{array}{l}\text { Median } \mathrm{T}: \mathrm{M}: 1.41 \text { (range, } 0.86-2 \text { ) } \\
\text { Median } \mathrm{T}: \mathrm{P}_{\text {mean }}: 0.96 \\
\text { (range, } 0.74-1.1 \text { ) } \\
\text { Median } \mathrm{T}: \mathrm{P}_{\max }: 1.29 \\
\text { (range, } 0.91-1.98 \text { ) }\end{array}$ & $T: P$ is good estimate of tumour hypoxia \\
\hline Lehtiö et al (2004) & ${ }^{18}$ F-FETNIM & $H \& N$ & 21 & $\begin{array}{l}\text { Median } T: P_{\text {max }}: 1.10 \\
\text { (range, } 0.81-1.98 \text { ) } \\
T: P>0.93 \text { used for hypoxic volume } \\
\text { definition }\end{array}$ & $\begin{array}{l}\text { Patients with higher fractional hypoxic volumes and } T: P \\
\text { correlated with poorer survival }\end{array}$ \\
\hline Hu et al (2013) & ${ }^{18}$ F-FETNIM & Lung & 42 & $\begin{array}{l}\text { SUV }_{\max , \text { Tumour: }}: 2.43 \\
\text { SUV }_{\max , \text { Normal }}: 0.87 \\
\mathrm{~T}: \mathrm{N}: 2.48 \text { at } 120 \text { min p.i. }\end{array}$ & $\begin{array}{l}\text { SUV }_{\max } \text { higher in tumours than in normal tissue. Similar } \\
\text { data observed at } 60 \text { and } 120 \mathrm{~min} \text { p.i. }\end{array}$ \\
\hline Li et al (2010) & ${ }^{18} \mathrm{~F}$-FETNIM & Lung & 26 & - & $\begin{array}{l}{ }^{18} \text { F-FETNIM T:B ratio and hypoxic volume were strong } \\
\text { predictors for overall survival. No correlation between } \\
{ }^{18} \text { F-FETNIM and }{ }^{18} \text { F-FDG uptake }\end{array}$ \\
\hline Vercellino et al (2012) & ${ }^{18}$ F-FETNIM & Cervical & 16 & $\mathrm{~T}: \mathrm{M}: 1.3-5.4$ & $\begin{array}{l}\text { High uptake associated with lower progression free and } \\
\text { overall survival }\end{array}$ \\
\hline Yue et al (2012) & ${ }^{18}$ F-FETNIM & Oesophageal & 28 & $\begin{array}{l}\text { SUV }_{\text {max, complete response }}: 3.2 \\
\text { SUV }_{\text {mean, complete response }}: 2.1 \\
\text { SUV }_{\text {max, partial response }}: 4.5 \\
\text { SUV }_{\text {mean, partial response }}: 2.9 \\
\text { SUV }_{\text {max, stable disease }}: 5.9 \\
\text { SUV } \\
\text { mean, stable disease: } 3.2 \\
\text { Threshold for hypoxia } \\
\text { SUV } \\
\text { max }\end{array}$ & $\begin{array}{l}\text { SUV } \\
\text { max } \\
\text { SUV }_{\text {max }} \text { associated with poor clinical response }\end{array}$ \\
\hline
\end{tabular}




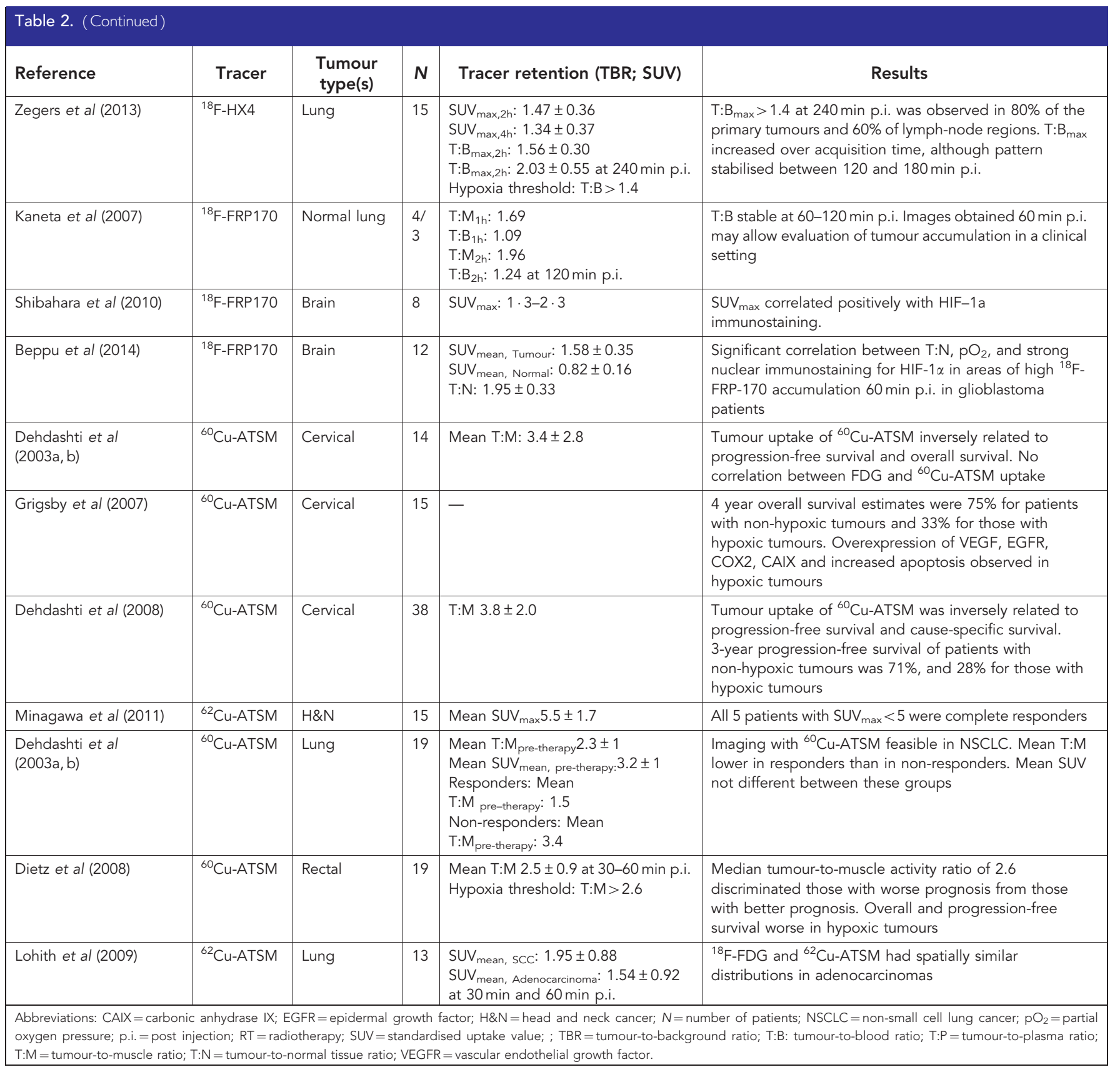

${ }^{18}$ F-fluoroerythronitroimidazole. ${ }^{18} \mathrm{~F}$-fluoroerythronitroimidazole $\left({ }^{18}\right.$ F-FETNIM) studies in head-and-neck (Lehtiö et al, 2001, 2003), lung (Li et al, 2010; Hu et al, 2013), and oesophageal cancer Yue et al (2012) calculated T:M in the range of $1.4-2.48$ at $2 \mathrm{~h}$ p.i. High tumour-to-muscle values were found to be indicative of reduced progression-free and overall survival in lung ( $\mathrm{Li}$ et al, 2010; $\mathrm{Hu}$ et al, 2013), head-and-neck (Lehtiö et al, 2004), oesophageal (Yue et al, 2012), and cervical (Vercellino et al, 2012) tumours. Clinical studies with ${ }^{18}$ F-FETNIM have been mainly carried out at the University of Turku, Finland. ${ }^{18}$ F-fluoroerythronitroimidazole is not being used at present in the United Kingdom or in the United States.

${ }^{18}$ F-RP-170. More recently, RP-170 (1-(2-1-(1H-methyl)ethoxy)methyl-2-nitroimidazole), another 2-nitroimidazole-based hypoxic radiosensitiser, has also been labelled with ${ }^{18} \mathrm{~F}$. The hypoxic selectivity of ${ }^{18} \mathrm{~F}$-FRP-170 was demonstrated in glioma patients on the basis of significant correlations between uptake, oxygen tension measurements and HIF-1 $\alpha$ immunostaining (Beppu et al, 2014). Studies in brain (Shibahara et al, 2010; Beppu et al, 2014) and lung (Kaneta et al, 2007) tumours indicated higher SUV for hypoxic than normal tissues; tumour-to-reference tissue ratio of 1.7 was calculated at $1 \mathrm{~h}$ p.i., which could be clinically sufficient for assessing hypoxia. The shorter interval before scanning, combined with improved hypoxic contrast compared with ${ }^{18} \mathrm{~F}$-FMISO, suggests that ${ }^{18}$ F-FRP-170 could potentially be useful in the clinic.

${ }^{18}$ F-HX4. ${ }^{18}$ F-3-fluoro-2-(4-((2-nitro-1H-imidazol-1-yl)methyl)$1 \mathrm{H}-1,2,3$-triazol-1-yl)propan-1-ol $\left({ }^{18} \mathrm{~F}-\mathrm{HX} 4\right)$ contains a 1,2,3anti-triazole moiety (as a synthetic convenience) rendering it more hydrophilic than ${ }^{18}$ F-FMISO. In head-and-neck tumours, ${ }^{18} \mathrm{~F}-\mathrm{HX} 4$ produced tumour-to-reference tissue values similar to ${ }^{18} \mathrm{~F}$-FMISO at relatively early time points p.i., indicating the potential advantage of shorter acquisition times (Chen et al, 2012). However, a more recent study in non-small-cell lung cancer (NSCLC) patients (Zegers et al, 2013) suggested that later scan times 


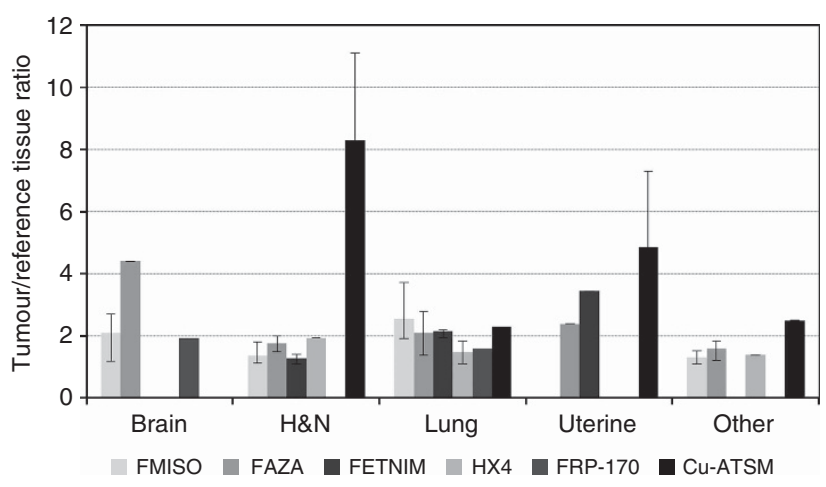

Figure 2. Tumour-to-reference tissue ratios and range in different tumour sites for the PET hypoxia tracers discussed in this review. For nitroimidazole-based analogues (FMISO, FAZA, FETNIM, HX4, FRP-170) values are given for acquisitions performed at $120 \mathrm{~min}$ post tracer administration. For Cu-ATSM, values are presented for scans conducted $60 \mathrm{~min}$.

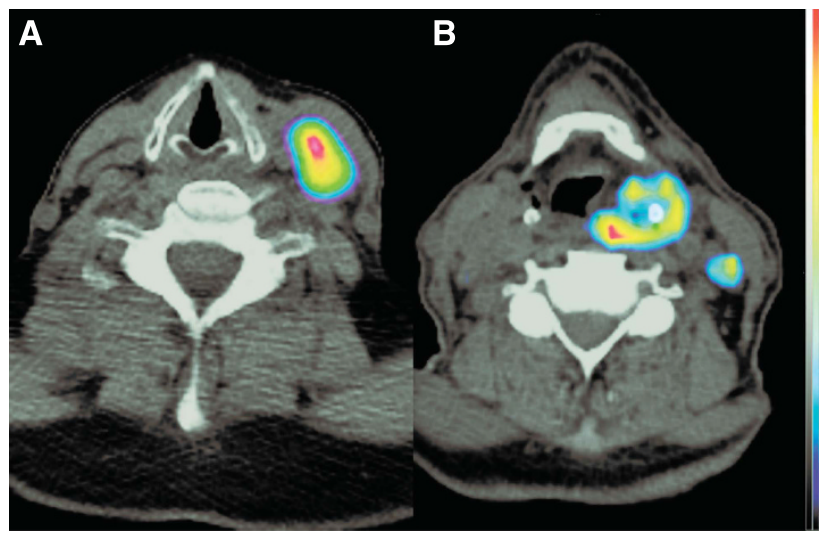

Figure 3. (A) Transverse ${ }^{18} \mathrm{~F}$-FMISO fused PET/CT overlay image acquired at baseline of a patient with metastatic renal cell carcinoma (mRCC) in the neck acquired at 2.5-3 h p.i (image courtesy of Professors Tim Eisen and Duncan Jodrell, University of Cambridge, UK). (B) ${ }^{64} \mathrm{Cu}$-ATSM fused PET/CT overlay image of a patient with advanced laryngeal squamous cell carcinoma (LSCC) at 80-90 min p.i. The transverse slice includes primary tumour and local lymph node (image courtesy of Dr Anastasia Chalkidou, King's College London, UK).

(2-4h p.i.) can further enhance the hypoxic-to-normoxic signal. In all of the above tracers, the more accurate hypoxic measure is made at least $2 \mathrm{~h}$ p.i., but the trade-off is the reduced radioactivity and noisier data.

\section{CU-DIACETYL-BIS(N ${ }^{4}$-METHYLTHIOSEMICARBAZONE)}

An alternative class of agents for the study of hypoxia with PET is based on a complex of $\mathrm{Cu}$ with diacetyl-bis $\left(N^{4}\right.$-methylthiosemicarbazone) (ATSM) ligands, among which ATSM is the prototype. Due to its lipophilicity and low molecular weight, Cu-ATSM is characterised by high membrane permeability and therefore rapid diffusion into cells. The hypoxic specificity of Cu-ATSM is thought to be partly imparted by the intracellular reduction of $\mathrm{Cu}$ (II) to $\mathrm{Cu}(\mathrm{I})$ combined with re-oxidation by intracellular molecular oxygen. Under hypoxic conditions, the unstable $\mathrm{Cu}(\mathrm{I})-\mathrm{ATSM}$ complex may further dissociate into $\mathrm{Cu}(\mathrm{I})$ and ATSM, leading to the intracellular trapping of the $\mathrm{Cu}(\mathrm{I})$ ion. In the presence of oxygen, the $[\mathrm{Cu}(\mathrm{I})-\mathrm{ATSM}]^{-}$can be re-oxidised to its parent compound, allowing efflux from the cell (Dearling and Packard, 2010).

Tumour-specific Cu-ATSM retention has been demonstrated for head-and-neck (Minagawa et al, 2011; Nyflot et al, 2012) (Figure 3B), lung (Takahashi et al, 2000; Dehdashti et al, 2003a, b; Lohith et al, 2009), cervical (Dehdashti et al, 2003a, b; Grigsby et al 2007; Lewis et al, 2008; Dehdashti et al, 2008), rectal tumours (Dietz et al, 2008) and gliomas (Tateishi et al, 2013). Hypoxia specificity may be dependent on tumour type: preclinical studies showed good correlation in the intratumour distribution of $\mathrm{Cu}-$ ATSM and ${ }^{18} \mathrm{~F}$-FMISO in a FaDu squamous carcinoma model but not at early time points in an R3327-AT anaplastic rat prostate tumour (O'Donoghue et al, 2005). A recent study has raised concerns about the hypoxic specificity of Cu-ATSM, as hepatic metabolism of the compound results in images that reflect the behaviour of ionic $\mathrm{Cu}$ (uptake of which may itself be hypoxiarelated) rather than Cu-ATSM itself, especially at later time points (1-24 h) (Hueting et al, 2014). Of concern is also the fact that while some preclinical studies show that tumour uptake of hypoxiaselective Cu-ATSM analogues (e.g., Cu-ATSE) decreases with increased oxygenation (McQuade et al, 2005), another report showed that increased oxygenation resulted in a decrease in uptake of FMISO, but not of Cu-ATSM (Matsumoto et al, 2007). Nevertheless, ${ }^{64} \mathrm{Cu}$-ATSM retention has been shown to correlate clinically with poor prognosis (Dehdashti et al, 2003a,b; 2008; Grigsby et al, 2007; Dietz et al, 2008). Attempts to investigate the relationship between the intratumoural distribution of Cu-ATSM with histological and other hypoxia markers have also yielded both positive and negative correlations. Although it appears to be premature to reject $\mathrm{Cu}-\mathrm{ATSM}$ on the grounds of hypoxic nonspecificity, further studies are required to elucidate the in vivo behaviour of this tracer to allow for better interpretation of the imaging information. The development of second-generation $\mathrm{Cu}$ ATSM analogues, with reduced lipophilicity and improved hypoxia selectivity and sensitivity, appears to be a promising alternative to Cu-ATSM (Handley et al, 2014). Cu-ATSM has several potential advantages relative to other tracers for the imaging of tumour hypoxia, including simpler synthesis/radiolabelling methodology and faster clearance from normoxic tissues, which allows shorter intervals between injection and imaging and higher hypoxic-tonormoxic contrast. Notwithstanding the limited availability of $\mathrm{Cu}$ isotopes, ${ }^{64} \mathrm{Cu}$-ATSM is currently being produced at a few research sites, and due to the 12-h half-life could potentially be utilised for clinical studies.

\section{CLINICAL APPLICATIONS OF PET HYPOXIA IMAGING}

Identification of tumour hypoxia and prediction of prognosis/ response to treatment. Identifying individuals with poor prognosis and those likely to benefit from hypoxia-targeted therapy are important objectives of PET hypoxia research. Several studies have shown that PET hypoxia imaging can provide information on prognosis. High ${ }^{18}$ F-FMISO retention has been associated with higher risk of loco-regional failure and shorter progression-free survival in head-and-neck (Rischin et al, 2006; Rajendran et al, 2006; Thorwarth et al, 2006; Dirix et al, 2009; Lee et al, 2009; Kikuchi et al, 2011) and renal cancer (Hugonet et al, 2011). Furthermore, a meta-review of the clinical data of over 300 patients concluded that FMISO is a predictor of poor treatment response and prognosis (Lee and Scott, 2007). Similar results have been reported for ${ }^{18}$ F-FETNIM in lung (Li et al, 2010), head-and-neck (Lehtiö et al, 2004), and oesophageal cancer (Yue et al, 2012), where high tumour-to-reference tissue values were also associated with poor patient outcomes. Studies conducted with ${ }^{18} \mathrm{~F}-\mathrm{FAZA}$ in 
Table 3. Matrix summarising clinical imaging findings with leading hypoxia tracers

\begin{tabular}{|c|c|c|c|c|c|c|c|}
\hline Tumour type & ${ }^{18} \mathrm{~F}-\mathrm{FMISO}$ & ${ }^{18} \mathrm{~F}-\mathrm{HX} 4$ & ${ }^{18} \mathrm{~F}-\mathrm{FAZA}$ & ${ }^{18} \mathrm{~F}$-FETNIM & ${ }^{18}$ F-EF5 & ${ }^{18}$ F-FRP170 & Cu-ATSM \\
\hline Brain & Yes & Not recommended & Yes & & Recommended & Yes & Recommended \\
\hline Head \& Neck & Yes & Yes & Yes & Yes & Yes & & Yes \\
\hline Breast & Yes & & & & & & \\
\hline Sarcoma & Variable data & & & & & & \\
\hline Lung & Yes & Yes & Yes & Yes & & Yes & Yes \\
\hline Lymphoma & & & Yes & & & & \\
\hline Renal & $\begin{array}{l}\text { Variable data } \\
\text { Not recommended }\end{array}$ & Not recommended & Not recommended & Not recommended & Not recommended & & Recommended \\
\hline Liver & Not recommended & Recommended & & Not recommended & Not recommended & & Not recommended \\
\hline Colorectal & Not recommended & & Yes & Not recommended & Not recommended & & Yes \\
\hline Bladder & Not recommended & Not recommended & Not recommended & Not recommended & Not recommended & & Recommended \\
\hline Cervical & & & Yes & Yes & & & Yes \\
\hline Prostate & & & No & & & & Not recommended \\
\hline
\end{tabular}

squamous cell carcinomas of the head and the neck (Mortensen et al, 2012) and Cu-ATSM in patients with cervical (Dehdashti et al, 2003a, b; Grigsby et al, 2007), lung (Dehdashti et al, 2003a, b), and rectal cancer (Dietz et al, 2008) have also demonstrated that lower tumour-to-muscle ratios are indicative of better prognosis, progression-free and overall survival. A meta-analysis of published PET hypoxia studies has demonstrated a common tendency towards poorer outcome in tumours showing higher tracer accumulation (Horsman et al, 2012). Decreased ${ }^{18}$ F-FMISO uptake in response to radio- or chemotherapy has been reported in brain (Swanson et al, 2009), head-and-neck (Yamane et al, 2011; Eschmann et al, 2007), lung (Koh et al, 1995; Gagel et al, 2006), and renal tumours (Hugonet et al, 2011); although some studies did not observe an analogous decrease with response to therapy (Thorwarth et al, 2006; Vera et al, 2011). Decreased tumour-tomuscle ratios signifying full or partial response to chemotherapy have also been obtained with Cu-ATSM in lung (Dehdashti et al, 2003a, b) and head-and-neck tumours (Minagawa et al, 2011), and ${ }^{18}$ F-FAZA in lung cancer (Trinkaus et al, 2013).

\section{RADIOTHERAPY PLANNING}

In oncology, there is interest in the identification of intratumoural areas with hypoxia to guide radiation dose escalation to radioresistant sub-volumes. Despite possible limitations associated with the reproducibility of hypoxic volume measurements (temporal changes and/or heterogeneity in the spatial distribution of intratumoural hypoxia), the biological information from PET hypoxia scans is being explored for the identification and delineation of hypoxic areas within the tumour mass for dose escalation. Modern radiation techniques, such as intensity modulated radiotherapy (IMRT) or image-guided radiotherapy (IGRT) can help with radiotherapy planning (Horsmann et al, 2012). 'Dose painting' by numbers, where a higher radiation dose is selectively delivered to areas of biological resistance identified either before or during the treatment course, has also been suggested (Geets et al, 2013). The feasibility of dose escalation to hypoxic sub-volumes has been primarily investigated in cancers of the head and neck, lung, and brain, and demonstrated with Cu-ATSM (Chao et al, 2001), ${ }^{18}$ F-FMISO (Lee et al, 2008), and ${ }^{18}$ F-FAZA (Grosu et al, 2007). Despite the fact that the majority of the aforementioned studies have not been conducted on actual patients, but on anthropomorphic phantoms (in silico)
(Rischin et al, 2006; Grosu et al, 2007; Lee et al, 2008), dose escalation on the basis of PET hypoxia imaging appears to be feasible, and further studies are required to investigate whether this can translate into clinical benefit.

\section{HYPOXIA THERAPEUTICS}

As the hypoxic microenvironment constitutes a unique characteristic of tumours, hypoxia can also be harnessed as a therapeutic target. The main strategies for targeting hypoxia involve hypoxic cell radiosensitisers (e.g., nimorazole), hypoxic cell cytotoxins (e.g., tirapazamine, TH-302, and PR-104A); and altering oxygen delivery (e.g., carbogen plus nicotinamide). Other approaches being investigated include hypoxia-selective gene therapy, altering metabolic pathways essential for survival under stress, and inhibitors of molecular targets activated in hypoxia (e.g., HIF-1) (Wilson and Hay, 2011). Imaging hypoxia with PET could facilitate the development of therapeutic agents by identifying patients with hypoxic tumours, and measuring response to hypoxia-modifying treatments providing a basis for individualising hypoxia-specific treatment, and/or assessing drug efficacy. Furthermore, it will allow development of new predictors and answer key questions, such as the relation of baseline or induced hypoxia to response to antiangiogenic drugs and the relation of baseline hypoxia to response to hypoxic-activated toxins. Such studies should be incorporated into trials of these agents routinely, to develop the necessary validation for their utility. This would greatly help the personalised and economic use of such therapies, which will be even more important if used in combination, for example, anti-angiogenics and hypoxia-activated toxins. The potential of PET hypoxia imaging in directing hypoxia therapeutics has been clinically demonstrated with tirapazamine with ${ }^{18} \mathrm{~F}$-FMISO in head and neck tumours, whereby only those with hypoxia benefited from bioreductive drugs (Rischin et al, 2006; Overgaard, 2011).

\section{CONSIDERATIONS}

The 'ideal' PET tracer for tumour hypoxia. Table 3 presents a summary of clinical imaging findings with the hypoxia tracers discussed in this review. None of the currently available tracers have all the properties that constitute the ideal PET hypoxia tracer, and therefore none is optimal for imaging hypoxia in all 
cancer types. Nevertheless, the feasibility of imaging hypoxia with PET has been clinically demonstrated in various tumour entities using several of the existing radiotracers. Much of the radiotracer selection stems from the availability of the tracer, ease of synthesis, and the tumour type.

The magnitude of the challenge of PET hypoxia imaging. A challenging aspect of PET hypoxia imaging is the fact that hypoxic tumours are often hypoperfused. Limited perfusion will restrict effective delivery of tracer into the tissue often, influencing tracer accumulation in regions of normal or tumour tissue, and often yielding results that are complex to interpret. Several studies have compared tumour perfusion with dynamic PET to ascertain whether tracer accumulation reflects blood flow during imaging. ${ }^{18} \mathrm{~F}$-FMISO (Bruehlmeier et al, 2004), ${ }^{18} \mathrm{~F}$-FETNIM (Lehtiö et al, 2001), and ${ }^{18}$ F-FAZA (Shi et al, 2010) exhibited similar distribution patterns to $\left[{ }^{15} \mathrm{O}\right]-\mathrm{H}_{2} \mathrm{O}$ PET (reflecting blood flow) up to 15 min p.i., while different patterns were observed at later imaging times, consistent with tracer accumulation in hypoxic regions. Pharmacokinetic analysis of ${ }^{18}$ F-FMISO data suggests that different hypoxia-perfusion profiles can be identified in tumours (Thorwarth et al, 2005); the latter perhaps corresponding with the heterogeneity observed in tumour hypoxia distribution patterns (Grosu et al, 2007). The significant heterogeneity of the tumour microenvironment in terms of perfusion and hypoxia necessitates further clinical studies, not only to evaluate hypoxia-perfusion patterns, but also their relationship to clinical outcome.

Validation of PET hypoxia measurements. Validation of PET tracers as indicators of regional hypoxia is extremely challenging and attempts to correlate PET images with other accepted hypoxia markers have produced mixed and contradictory results. While oxygen electrodes are considered to be the gold standard against which PET hypoxia measurements are authenticated, comparisons may yield several discrepancies due to the sampling limitations of oxygen probes and the fact that it measures hypoxia in a different location (interstitial for oxygen probes $v s$ intracellular for PET), as well as the fact that this technique will fail to distinguish between necrotic and viable hypoxic tissue (Höckel et al, 1993). This may partly explain results from several studies that have reported mixed correlations between tracer uptake and oxygen electrode measurements in various tumour types (Bentzen et al, 2003; Gagel et al, 2004, 2007; Zimny et al, 2006; Mortensen et al, 2010). Indirect immunohistochemical methods based on the detection of exogenous (e.g., pimonidazole and EF5) or endogenous hypoxia markers (e.g., CAIX and HIF-1) have also been employed (Dehdashti et al, 2003a, b; Jubb et al, 2010), albeit with limited success. This is primarily due to the fact that comparisons as such rely on reproducible staining, and several representative biopsies (which are not always available), and may often require a technically challenging spatial co-registration between PET images with immunohistochemistry photographs for analogies to be drawn. Of note is the fact that although tracer accumulation has been widely compared with pimonidazole staining preclinically (Dubois et al, 2004), equivalent clinical comparisons have not yet been performed. The differential detection of acute and chronic hypoxia and the discrepancy between hypoxia at the microscopic level and the macroscopic resolution of the PET voxel are factors that will also limit the accuracy of such comparisons (Mortensen et al, 2010).

Reproducibility of PET hypoxia measurements. Validation of the reproducibility of PET hypoxia measurements is also particularly important for clinical applications. There are limited clinical data available on scan reproducibility with PET hypoxia biomarkers. Studies with ${ }^{18}$ F-FMISO in head-and-neck cancer reported reproducible hypoxic volumes in PET scans performed
3 days apart, but a considerable degree of intratumoural spatial variability in tracer accumulation (Nehmeh et al, 2008). Another study with ${ }^{18} \mathrm{~F}$-FMISO in lung cancer showed good inter-observer reproducibility on the basis of visual analysis, but low interobserver agreement with respect to hypoxic volume measurements (Thureau et al, 2013). A more recent ${ }^{18}$ F-FMISO study in headand-neck cancer reported high reproducibility in SUV and tumour-to-reference tissue measurements in scans acquired 2 days apart (Okamoto et al, 2013). Other than ${ }^{18} \mathrm{~F}$-FMISO, a study with ${ }^{18}$ F-FETNIM in oesophageal cancer patients observed similar uptake values between scans performed on separate days before concurrent chemoradiotherapy, but a shift in the geographical location of hypoxic regions (Yue et al, 2012). These heterogeneous findings can be partly explained by the dynamic character of hypoxia that will limit scan reproducibility. Although acute hypoxia has been shown to minimally influence ${ }^{18}$ F-FMISO PET imaging in simulations (Mönnich et al, 2012), a study in head-andneck tumours that used sequential ${ }^{18} \mathrm{~F}$-FMISO scans to distinguish between regions of acute and chronic hypoxia, accounted for $14-52 \%$ of acute hypoxia (Wang et al, 2009); a percentage that is comparable to the proportion of acute hypoxia measured in rodent tumours. Methodological discrepancies (scan setup and image acquisition protocol), the selection of hypoxic-to-normoxic thresholds for the definition of hypoxic regions, the temporal variability in intratumoural $\mathrm{pO}_{2}$ levels between consecutive measurements, as well as the small number of patients in the majority of the studies may also account for the observed disparities in reproducibility. Further studies addressing the variability of PET hypoxia measurements are warranted, so as to clarify uncertainties in tumour hypoxia quantification.

\section{CONCLUSIONS}

As a number of PET hypoxia tracers have now been evaluated in cancer patients, it is apparent that PET imaging can be a powerful tool to identify hypoxia in the clinical setting. Although none of the currently available tracers exhibit all of the properties of the 'ideal' hypoxia tracer or are optimal for imaging hypoxia in all tumour types, studies have demonstrated the feasibility for imaging hypoxia in various cancers. As the clinical utility and limitations of PET hypoxia biomarkers are now being elucidated the process will be facilitated by performing larger studies with these tracers using standardised protocols and hypoxia definitions so as to improve comparison between tracers in various tumour types. This may be best achieved via inter-institutional collaborations that should help to advance study designs and homogeneous data reporting. Equally important are the performance of testretest studies, harmonisation of data reporting, and clinical validation of hypoxia tracers. These key objectives must be addressed before PET hypoxia tracers can be used to their full clinical utility.

Search strategy and selection criteria. We searched PubMed and Scopus using combinations of the following search terms: 'tumor hypoxia', 'oncology', 'PET', 'positron emission tomography', radiotherapy', 'nitroimidazoles', 'fluoromisonidazole', 'pimonidazole', 'FMISO', 'FAZA', 'FETNIM', 'FRP-170', 'HX4', 'Cu-ATSM'. The search results were screened for relevance and the reference lists of relevant publications were also surveyed. PubMed and Scopus article recommendations were also examined for relevance. Only papers published in English were considered. The final reference list was compiled by considering papers published between January 1973 and May 2014. 


\section{ACKNOWLEDGEMENTS}

Cancer Research UK (CRUK) funded the National Cancer Research Institute (NCRI) PET Research Working party to organise a meeting to discuss imaging cancer with hypoxia tracers and Positron Emission Tomography. IF was funded by CRUK and is also supported by the Chief Scientific Office. ALH is supported by CRUK and the Breast Cancer Research Foundation. RM is funded by NIHR Cambridge Biomedical Research Centre. We would also like to thank Professors Tim Eisen and Duncan Jodrell, University of Cambridge, UK and Dr Anastasia Chalkidou, King's College London, UK for providing the ${ }^{18} \mathrm{~F}-\mathrm{FMISO}$ and ${ }^{64} \mathrm{Cu}-$ ATSM images illustrated in this review.

\section{CONFLICT OF INTEREST}

The authors declare no conflict of interest.

\section{AUTHOR CONTRIBUTIONS}

INF contributed organisation of the hypoxia workshop, literature search and wrote core manuscript and edited various versions of manuscript, approved final version of the manuscript. RM contributed to literature search, edited manuscript, prepared Figure 2, approved final version of the manuscript. PJB attended the hypoxia workshop, wrote Cu-ATSM section and edited various versions of manuscript, approved final version of the manuscript. CW attended the hypoxia workshop, wrote radiotherapy section, approved final version of the manuscript. KJW attended the hypoxia workshop, wrote therapeutics section, approved final version of the manuscript. ALH attended the hypoxia workshop, wrote section on tumour hypoxia, approved final version of the manuscript. JD attended the hypoxia workshop, prepared Figure 1, approved final version of the manuscript. SL attended the hypoxia workshop, contributed to tumour hypoxia section, approved final version of the manuscript. CB attended the hypoxia workshop, $\mathrm{Cu}$-ATSM section, approved final version of the manuscript. FJG concept of the review, organisation of the hypoxia workshop, editing of various versions of the manuscript, final approval of the manuscript.

\section{REFERENCES}

Abolmaali N, Haase R, Koch A, Zips D, Steinbach J, Baumann M, Kotzerke J, Zöphel K (2011) Two or four hour $\left[{ }^{18} \mathrm{~F}\right]$ FMISO-PET in HNSCC: When is the contrast best? Nuklearmedizin 50(1): 22-27.

Bentzen L, Keiding S, Nordsmark M, Falborg L, Hansen SB, Keller J, Nielsen OS, Overgaard J (2003) Tumour oxygenation assessed by ${ }^{18}$ F-fluoromisonidazole PET and polarographic needle electrodes in human soft tissue tumours. Radiother Oncol 67(3): 339-344.

Beppu T, Terasaki K, Sasaki T, Fujiwara S, Matsuura H, Ogasawara K, Sera K, Yamada N, Uesugi N, Sugai T, Kudo K, Sasaki M, Ehara S, Iwata R, Takai Y (2014) Standardized uptake value in high uptake area on positron emission tomography with ${ }^{18} \mathrm{~F}$-FRP170 as a hypoxic cell tracer correlates with intratumoral oxygen pressure in glioblastoma. Mol Imaging Biol 16(1): 1-9.

Bollineni VR, Kerner GSMA, Pruim J, Steenbakkers RJ, Wiegman EM, Koole MJ, de Groot EH, Willemsen AT, Luurtsema G, Widder J, Groen HJ, Langendijk JA (2013) PET imaging of tumor hypoxia using ${ }^{18} \mathrm{~F}$-fluoroazomycin arabinoside in stage III-IV non-small cell lung cancer patients. J Nucl Med 54(8): 1175-1180.

Bristow RG, Hill RP (2008) Hypoxia and metabolism: Hypoxia, DNA repair and genetic instability. Nat Rev Cancer 8(3): 180-192.

Bruehlmeier M, Roelcke U, Schubiger PA, Ametamey SM (2004) Assessment of hypoxia and perfusion in human brain tumors using PET with ${ }^{18}$ F-fluoromisonidazole and ${ }^{15} \mathrm{O}-\mathrm{H}_{2} \mathrm{O}$. J Nucl Med 45(11): 1851-1859.
Chao KSC, Bosch WR, Mutic S, Lewis JS, Dehdashti F, Mintun MA, Dempsey JF, Perez CA, Purdy JA, Welch MJ (2001) A novel approach to overcome hypoxic tumor resistance: Cu-ATSM-guided intensitymodulated radiation therapy. Int J Radiat Oncol Biol Phys 49(4): 1171-1182.

Chapman JD (1979) Hypoxic sensitisers - Implications for radiation therapy. N Engl J Med 301(26): 1429-1432.

Chen L, Zhang Z, Kolb HC, Walsh JC, Zhang J, Guan Y (2012) ${ }^{18}$ F-HX4 hypoxia imaging with PET/CT in head and neck cancer: A comparison with ${ }^{18}$ F-FMISO. Nucl Med Commun 33(10): 1096-1102.

Cheng J, Lei L, Xu J, Sun Y, Zhang Y, Wang X, Pan L, Shao Z, Zhang Y, Liu G (2013) ${ }^{18}$ F-fluoromisonidazole PET/CT: a potential tool for predicting primary endocrine therapy resistance in breast cancer. J Nucl Med 54(3): 333-340.

Cher LM, Murone C, Lawrentschuk N, Ramdave S, Papenfuss A, Hannah A, O’Keefe GJ, Sachinidis JI, Berlangieri SU, Fabinyi G, Scott AM (2006) Correlation of hypoxic cell fraction and angiogenesis with glucose metabolic rate in gliomas using ${ }^{18} \mathrm{~F}$-fluoromisonidazole, ${ }^{18} \mathrm{~F}$-FDG PET, and immunohistochemical studies. J Nucl Med 47(3): 410-418.

Cherk MH, Foo SS, Poon AMT, Knight SR, Murone C, Papenfuss AT, Sachinidis JI, Saunder TH, O'Keefe GJ, Scott AM (2006) Lack of correlation of hypoxic cell fraction and angiogenesis with glucose metabolic rate in non-small cell lung cancer assessed by ${ }^{18}$ F-fluoromisonidazole and ${ }^{18}$ F-FDG PET. J Nucl Med 47(12): 1921-1926. Dearling JLJ, Packard AB (2010) Some thoughts on the mechanism of cellular trapping of $\mathrm{Cu}(\mathrm{II})-\mathrm{ATSM}$. Nucl Med Biol 37(3): 237-243.

Dehdashti F, Grigsby PW, Mintun MA, Lewis JS, Siegel BA, Welch MJ (2003a) Assessing tumor hypoxia in cervical cancer by positron emission tomography with ${ }^{60} \mathrm{Cu}$-ATSM: Relationship to therapeutic response a preliminary report. Int J Radiat Oncol Biol Phys 55(5): 1233-1238.

Dehdashti F, Mintun MA, Lewis JS, Bradley J, Govindan R, Laforest R, Welch MJ, Siegel BA (2003b) In vivo assessment of tumor hypoxia in lung cancer with ${ }^{60} \mathrm{Cu}-\mathrm{ATSM}$. Eur J Nucl Med Mol Imaging 30(6): 844-850.

Dehdashti F, Grigsby PW, Lewis JS, Laforest R, Siegel BA, Welch MJ (2008) Assessing tumor hypoxia in cervical cancer by PET with ${ }^{60} \mathrm{Cu}$-labeled diacetyl-bis(N4-methylthiosemicarbazone). J Nucl Med 49(2): 201-205.

Dietz DW, Dehdashti F, Grigsby PW, Malyapa RS, Myerson RJ, Picus J, Ritter J, Lewis JS, Welch MJ, Siegel BA (2008) Tumor hypoxia detected by positron emission tomography with ${ }^{60} \mathrm{Cu}-\mathrm{ATSM}$ as a predictor of response and survival in patients undergoing neoadjuvant chemoradiotherapy for rectal carcinoma: a pilot study. Dis Colon Rectum 51(11): 1641-1648.

Dirix P, Vandecaveye V, De Keyzer F, Stroobants S, Hermans R, Nuyts S (2009) Dose painting in radiotherapy for head and neck squamous cell carcinoma: value of repeated functional imaging with ${ }^{18}$ F-FDG PET, ${ }^{18}$ F-fluoromisonidazole PET, diffusion-weighted MRI, and dynamic contrast-enhanced MRI. J Nucl Med 50(7): 1020-1027.

Dubois L, Landuyt W, Haustermans K, Dupont P, Bormans G, Vermaelen P, Flamen P, Verbeken E, Mortelmans L (2004) Evaluation of hypoxia in an experimental rat tumour model by $\left[{ }^{18} \mathrm{~F}\right]$ Fluoromisonidazole PET and immunohistochemistry. Br J Cancer 91(11): 1947-1954.

Eschmann SM, Paulsen F, Bedeshem C, Machulla HJ, Hehr T, Bamberg M, Bares R (2007) Hypoxia--imaging with ${ }^{18}$ F-Misonidazole and PET: changes of kinetics during radiotherapy of head-and-neck cancer. Radiother Oncol 83(3): 406-410.

Gagel B, Reinartz P, DiMartino E, Zimny M, Pinkawa M, Maneschi P, Stanzel S, Hamacher K, Coenen HH, Westhofen M, Büll U, Eble MJ (2004) pO2 polarography versus positron emission tomography ( $\left[{ }^{18} \mathrm{~F}\right]$ fluoromisonidazole, $\left[{ }^{18} \mathrm{~F}\right]$-2-fluoro- $2^{\prime}$-deoxyglucose): an appraisal of radiotherapeutically relevant hypoxia. Strahlenther Onkol 180(10): 616-622.

Gagel B, Reinartz P, Demirel C, Kaiser HJ, Zimny M, Piroth M, Pinkawa M, Stanzel S, Asadpour B, Hamacher K, Coenen HH, Buell U, Eble MJ (2006) $\left[{ }^{18} \mathrm{~F}\right]$-fluromisonidazole and $\left[{ }^{18} \mathrm{~F}\right]$ fluorodeoxyglucose positron emission tomography in response evaluation after chemo-/radiotherapy of non-small-cell lung cancer: a feasibility study. BMC Cancer 6: 51.

Gagel B, Piroth M, Pinkawa M, Reinartz P, Zimny M, Kaiser HJ, Stanzel S, Asadpour B, Demirel C, Hamacher K, Coenen HH, Scholbach T, Maneschi P, DiMartino E, Eble MJ (2007) pO polarography, contrast enhanced color duplex sonography (CDS), $\left[{ }^{18} \mathrm{~F}\right]$ fluoromisonidazole and $\left[{ }^{18} \mathrm{~F}\right]$ fluorodeoxyglucose positron emission tomography: validated methods for the evaluation of therapy-relevant tumor oxygenation or only bricks in the puzzle of tumor hypoxia? BMC Cancer 7: 113. 
Garcia-Parra R, Wood D, Shah RB, Siddiqui J, Hussain H, Park H, Desmond T, Meyer C, Piert M (2011) Investigation on tumor hypoxia in resectable primary prostate cancer as demonstrated by ${ }^{18} \mathrm{~F}$-FAZA PET/CT utilizing multimodality fusion techniques. Eur J Nucl Med Mol Imaging 2011: $1-8$.

Geets X, Grégoire V, Lee JA (2013) Implementation of hypoxia PET imaging in radiation therapy planning. Q J Nucl Med Mol Imaging 57(3): 271-282.

Grigsby PW, Malyapa RS, Higashikubo R, Schwarz JK, Welch MJ, Huettner PC, Dehdashti F (2007) Comparison of molecular markers of hypoxia and imaging with ${ }^{60} \mathrm{Cu}$-ATSM in cancer of the uterine cervix. Mol Imaging Biol 9(5): 278-283.

Grosu AL, Souvatzoglou M, Röper B, Dobritz M, Wiedenmann N, Jacob V, Wester HJ, Reischl G, Machulla HJ, Schwaiger M, Molls M, Piert M (2007) Hypoxia imaging with FAZA-PET and theoretical considerations with regard to dose painting for individualization of radiotherapy in patients with head and neck cancer. Int J Radiat Oncol Biol Phys 69(2): 541-551.

Handley MG, Medina RA, Mariotti E, Mariotti E, Kenny GD, Shaw KP, Yan R, Eykyn TR, Blower PJ, Southworth R (2014) Cardiac hypoxia imaging: second-generation analogues of ${ }^{64} \mathrm{Cu}-\mathrm{ATSM}$. J Nucl Med 55(3): 488-494.

Havelund BM, Holdgaard PC, Rafaelsen SR, Mortensen LS, Theil J, Bender D, Pløen J, Spindler KL, Jakobsen A (2013) Tumour hypoxia imaging with ${ }^{18} \mathrm{~F}$-fluoroazomycinarabinofuranoside PET/CT in patients with locally advanced rectal cancer. Nucl Med Commun 34(2): 155-161.

Hicks RJ, Rischin D, Fisher R, Binns D, Scott AM, Peters LJ (2005) Utility of FMISO PET in advanced head and neck cancer treated with chemoradiation incorporating a hypoxia-targeting chemotherapy agent. Eur J Nucl Med Mol Imaging 32(12): 1384-1391.

Höckel M, Knoop C, Schlenger K, Vorndran B, Baussmann E, Mitze M, Knapstein PG, Vaupel $\mathrm{P}$ (1993) Intratumoral $\mathrm{pO}_{2}$ predicts survival in advanced cancer of the uterine cervix. Radiother Oncol 26(1): 45-50.

Horsman MR, Mortensen LS, Petersen JB, Busk M, Overgaard J (2012) Imaging hypoxia to improve radiotherapy outcome. Nat Rev Clin Oncol 9(12): 674-687.

Hu M, Xing L, Mu D, Yang W, Yang G, Kong L, Yu J (2013) Hypoxia imaging with ${ }^{18} \mathrm{~F}$-fluoroerythronitroimidazole integrated PET/CT and immunohistochemical studies in non-small cell lung cancer. Clin Nucl Med 38(8): 591-596.

Hueting R, Kersemans V, Cornelissen B, Tredwell M, Hussien K, Christlieb M, Gee AD, Passchier J, Smart SC, Dilworth JR, Gouverneur V, Muschel RJ (2014) A comparison of the behavior of ${ }^{64} \mathrm{Cu}$-acetate and ${ }^{64} \mathrm{Cu}$-ATSM in vitro and in vivo. J Nucl Med 55(1): 128-134.

Hugonnet F, Fournier L, Medioni J, Smadja C, Hindié E, Huchet V, Itti E, Cuenod CA, Chatellier G, Oudard S, Faraggi M. Hypoxia in Renal Cancer Multicenter Group (2011) Metastatic renal cell carcinoma: relationship between initial metastasis hypoxia, change after 1 month's sunitinib, and therapeutic response: an ${ }^{18} \mathrm{~F}$-fluoromisonidazole PET/CT study. J Nucl Med 52(7): 1048-1055.

Jubb AM, Buffa FM, Harris AL (2010) Assessment of tumour hypoxia for prediction of response to therapy and cancer prognosis. J Cell Mol Med 14(1-2): 18-29.

Kaneta T, Takai Y, Iwata R, Hakamatsuka T, Yasuda H, Nakayama K, Ishikawa Y, Watanuki S, Furumoto S, Funaki Y, Nakata E, Jingu K, Tsujitani M, Ito M, Fukuda H, Takahashi S, Yamada S (2007) Initial evaluation of dynamic human imaging using ${ }^{18} \mathrm{~F}-\mathrm{FRP} 170$ as a new PET tracer for imaging hypoxia. Ann Nucl Med 21(2): 101-107.

Kikuchi M, Yamane T, Shinohara S, Fujiwara K, Hori SY, Tona Y, Yamazaki H, Naito Y, Senda M (2011) ${ }^{18} \mathrm{~F}$-fluoromisonidazole positron emission tomography before treatment is a predictor of radiotherapy outcome and survival prognosis in patients with head and neck squamous cell carcinoma. Ann Nucl Med 25(9): 625-633.

Koh WJ, Bergman KS, Rasey JS, Peterson LM, Evans ML, Graham MM, Grierson JR, Lindsley KL, Lewellen TK, Krohn KA, Griffin TW (1995) Evaluation of oxygenation status during fractionated radiotherapy in human nonsmall cell lung cancers using [F-18]fluoromisonidazole positron emission tomography. Int J Radiat Oncol Biol Phys 33(2): 391-398.

Lee NY, Mechalakos JG, Nehmeh S, Lin Z, Squire OD, Cai S, Chan K, Zanzonico PB, Greco C, Ling CC, Humm JL, Schöder H (2008) Fluorine-18-labeled fluoromisonidazole positron emission and computed tomography-guided intensity-modulated radiotherapy for head and neck cancer: a feasibility study. Int J Radiat Oncol Biol Phys 70(1): 2-13.

Lee N, Nehmeh S, Schöder H, Fury M, Chan K, Ling CC, Humm J (2009) Prospective trial incorporating pre-/mid-treatment $\left[{ }^{18} \mathrm{~F}\right]$-misonidazole positron emission tomography for head-and-neck cancer patients undergoing concurrent chemoradiotherapy. Int J Radiat Oncol Biol Phys 75(1): 101-108.

Lee ST, Scott AM (2007) Hypoxia positron emission tomography imaging with ${ }^{18}$ F-fluoromisonidazole. Semin Nucl Med 37(6): 451-461.

Lehtiö K, Oikonen V, Grönroos T, Eskola O, Kalliokoski K, Bergman J, Solin O, Grénman R, Nuutila P, Minn H (2001) Imaging of blood flow and hypoxia in head and neck cancer: initial evaluation with $\left[{ }^{15} \mathrm{O}^{1} \mathrm{H}_{2} \mathrm{O}\right.$ and $\left[{ }^{18}\right.$ F]Fluoroerythronitroimidazole PET. J Nucl Med 42(11): 1643-1652.

Lehtiö K, Oikonen V, Nyman S, Grönroos T, Roivainen A, Eskola O, Minn H (2003) Quantifying tumour hypoxia with fluorine-18-

fluoroerythronitroimidazole $\left(\left[{ }^{18} \mathrm{~F}\right] \mathrm{FETNIM}\right)$ and PET using the tumour to plasma ratio. Eur J Nucl Med Mol Imaging 30(1): 101-108.

Lehtiö K, Eskola O, Viljanen T, Oikonen V, Grönroos T, Sillanmäki L, Grénman R, Minn H (2004) Imaging perfusion and hypoxia with PET to predict radiotherapy response in head-and-neck cancer. Int $J$ Radiat Oncol Biol Phys 59(4): 971-982.

Lewis JS, Laforest R, Dehdashti F, Grigsby PW, Welch MJ, Siegel BA (2008) An imaging comparison of ${ }^{64} \mathrm{Cu}$-ATSM and ${ }^{60} \mathrm{Cu}$-ATSM in cancer of the uterine cervix. J Nucl Med 49(7): 1177-1182.

Li L, Hu M, Zhu H, Zhao W, Yang G, Yu J (2010) Comparison of ${ }^{18} \mathrm{~F}$-fluoroerythronitroimidazole and ${ }^{18} \mathrm{~F}$-fluorodeoxyglucose positron emission tomography and prognostic value in locally advanced non-smallcell lung cancer. Clin Lung Cancer 11(5): 335-340.

Lohith TG, Kudo T, Demura Y, Umeda Y, Kiyono Y, Fujibayashi Y, Okazawa $\mathrm{H}$ (2009) Pathophysiologic correlation between ${ }^{62} \mathrm{Cu}-\mathrm{ATSM}$ and ${ }^{18} \mathrm{~F}$-FDG in lung cancer. J Nucl Med 50(12): 1948-1953.

Matsumoto K, Szajek L, Krishna MC, Cook JA, Seidel J, Grimes K, Carson J, Sowers AL, English S, Green MV, Bacharach SL, Eckelman WC, Mitchell JB (2007) The influence of tumor oxygenation on hypoxia imaging in murine squamous cell carcinoma using $\left[{ }^{64} \mathrm{Cu}\right] \mathrm{Cu}-\mathrm{ATSM}$ or $\left[{ }^{18}\right.$ F]Fluoromisonidazole positron emission tomography. Int J Oncol 30(4): 873-881.

McQuade P, Martin KE, Castle TC, Went MJ, Blower PJ, Welch MJ, Lewis JS (2005) Investigation into ${ }^{64} \mathrm{Cu}$-labeled Bis(selenosemicarbazone) and Bis(thiosemicarbazone) complexes as hypoxia imaging agents. Nucl Med Biol 32(2): 147-156.

Minagawa Y, Shizukuishi K, Koike I, Horiuchi C, Watanuki K, Hata M, Omura M, Odagiri K, Tohnai I, Inoue T, Tateishi U (2011) Assessment of tumor hypoxia by ${ }^{62} \mathrm{Cu}-\mathrm{ATSM}$ PET/CT as a predictor of response in head and neck cancer: a pilot study. Ann Nucl Med 25(5): 339-345.

Mönnich D, Troost EGC, Kaanders JHAM, Oyen WJG, Alber M, Thorwarth D (2012) Modelling and simulation of the influence of acute and chronic hypoxia on $\left[{ }^{18} \mathrm{~F}\right]$ fluoromisonidazole PET imaging. Phys Med Biol 57(6): 1675-1684.

Mortensen LS, Buus S, Nordsmark M, Bentzen L, Munk OL, Keiding S, Overgaard J (2010) Identifying hypoxia in human tumors: a correlation study between ${ }^{18} \mathrm{~F}$-FMISO PET and the Eppendorf oxygen-sensitive electrode. Acta Oncol 49(7): 934-940.

Mortensen LS, Johansen J, Kallehauge J, Primdahl H, Busk M, Lassen P, Alsner J, Sørensen BS, Toustrup K, Jakobsen S, Petersen J, Petersen H, Theil J, Nordsmark M, Overgaard J (2012) FAZA PET/CT hypoxia imaging in patients with squamous cell carcinoma of the head and neck treated with radiotherapy: results from the DAHANCA 24 trial. Radiother Oncol 105(1): 14-20.

Nehmeh SA, Lee NY, Schröder H, Squire O, Zanzonico PB, Erdi YE, Greco C, Mageras G, Pham HS, Larson SM, Ling CC, Humm JL (2008) Reproducibility of intratumor distribution of ${ }^{18} \mathrm{~F}$-fluoromisonidazole in head and neck cancer. Int J Radiat Oncol Biol Phys 70(1): 235-242.

Nyflot MJ, Harari PM, Yip S, Perlman SB, Jeraj R (2012) Correlation of PET images of metabolism, proliferation and hypoxia to characterize tumor phenotype in patients with cancer of the oropharynx. Radiother Oncol 105(1): 36-40.

O’Donoghue JA, Zanzonico P, Pugachev A, Wen B, Smith-Jones P, Cai S, Burnazi E, Finn RD, Burgman P, Ruan S, Lewis JS, Welch MJ, Ling CC, Humm JL (2005) Assessment of regional tumor hypoxia using ${ }^{18} \mathrm{~F}$-fluoromisonidazole and ${ }^{64} \mathrm{Cu}(\mathrm{II})$-diacetyl-bis(N4methylthiosemicarbazone) positron emission tomography: comparative study featuring microPET imaging, $\mathrm{pO}_{2}$ probe measurement, autoradiography, and fluorescent microscopy in the R3327-AT and FaDu rat tumor models. Int J Radiat Oncol Biol Phys 61(5): 1493-1502.

Okamoto S, Shiga T, Yasuda K, Ito YM, Magota K, Kasai K, Kuge Y, Shirato H, Tamaki N (2013) High reproducibility of tumor hypoxia 
evaluated by ${ }^{18} \mathrm{~F}$-fluoromisonidazole pet for head and neck cancer. J Nucl Med 54(2): 201-207.

Overgaard J (2011) Hypoxic modification of radiotherapy in squamous cell carcinoma of the head and neck - a systematic review and meta-analysis. Radiother Oncol 100(1): 22-32.

Postema EJ, McEwan AJB, Riauka TA, Kumar P, Richmond DA, Abrams DN, Wiebe LI (2009) Initial results of hypoxia imaging using 1- $\alpha$-d-(5-deoxy5 - $\left[{ }^{18} \mathrm{~F}\right]$-fluoroarabinofuranosyl)-2-nitroimidazole $\left({ }^{18} \mathrm{~F}\right.$-FAZA). Eur J Nucl Med Mol Imaging 36(10): 1565-1573.

Rajendran JG, Wilson DC, Conrad EU, Peterson LM, Bruckner JD, Rasey JS, Chin LK, Hofstrand PD, Grierson JR, Eary JF, Krohn KA (2003) $\left[{ }^{18} \mathrm{~F}\right]$ FMISO and $\left[{ }^{18} \mathrm{~F}\right]$ FDG PET imaging in soft tissue sarcomas: correlation of hypoxia, metabolism and VEGF expression. Eur J Nucl Med Mol Imaging 30(5): 695-704.

Rajendran JG, Mankoff DA, O'Sullivan F, Peterson LM, Schwartz DL, Conrad EU, Spence AM, Muzi M, Farwell DG, Krohn KA (2004) Hypoxia and glucose metabolism in malignant tumors: evaluation by

$\left[{ }^{18} \mathrm{~F}\right]$ Fluoromisonidazole and $\left.\left[{ }^{18} \mathrm{~F}\right]\right]$ Fluorodeoxyglucose positron emission tomography imaging. Clin Cancer Res 10(7): 2245-2252.

Rajendran JG, Schwartz DL, O'Sullivan J, Peterson LM, Ng P, Scharnhorst J, Grierson JR, Krohn KA (2006) Tumor hypoxia imaging with [F-18] fluoromisonidazole positron emission tomography in head and neck cancer. Clin Cancer Res 12(18): 5435-5441.

Rasey JS, Koh WJ, Evans ML, Peterson LM, Lewellen TK, Graham MM, Krohn KA (1996) Quantifying regional hypoxia in human tumors with positron emission tomography of $\left[{ }^{18} \mathrm{~F}\right]$ fluoromisonidazole: a pretherapy study of 37 patients. Int J Radiat Oncol Biol Phys 36(2): 417-428.

Rischin D, Hicks RJ, Fisher R, Binns D, Corry J, Porceddu S, Peters LJ (2006) Prognostic significance of $\left[{ }^{18} \mathrm{~F}\right]$-misonidazole positron emission tomography-detected tumor hypoxia in patients with advanced head and neck cancer randomly assigned to chemoradiation with or without tirapazamine: a substudy of Trans-Tasman Radiation Oncology Group study 98.02. J Clin Oncol 24(13): 2098-2104.

Roels S, Slagmolen P, Nuyts J, Lee JA, Loeckx D, Maes F, Stroobants S, Penninckx F, Haustermans K (2008) Biological image-guided radiotherapy in rectal cancer: Is there a role for FMISO or FLT, next to FDG? Acta Oncol 47(7): 1237-1248.

Sato J, Kitagawa Y, Yamazaki Y, Hata H, Okamoto S, Shiga T, Shindoh M, Kuge Y, Tamaki N (2013) ${ }^{18}$ F-fluoromisonidazole PET uptake is correlated with hypoxia-inducible factor-1a expression in oral squamous cell carcinoma. J Nucl Med 54(7): 1060-1065.

Schuetz M, Schmid MP, Pötter R, Kommata S, Georg D, Lukic D, Dudczak R, Kletter K, Dimopoulos J, Karanikas G, Bachtiary B (2010) Evaluating repetitive ${ }^{18} \mathrm{~F}$-fluoroazomycin-arabinoside $\left({ }^{18} \mathrm{FAZA}\right)$ PET in the setting of MRI guided adaptive radiotherapy in cervical cancer. Acta Oncol 49(7): 941-947.

Segard T, Robins PD, Yusoff IF, Ee H, Morandeau L, Campbell EM, Francis RJ (2013) Detection of hypoxia with ${ }^{18} \mathrm{~F}$-fluoromisonidazole $\left({ }^{18} \mathrm{~F}\right.$-FMISO) $\mathrm{PET} / \mathrm{CT}$ in suspected or proven pancreatic cancer. Clin Nucl Med 38(1): $1-6$.

Semenza GL (2004) Hydroxylation of HIF-1: oxygen sensing at the molecular level. Physiology 19(4): 176-182.

Shi K, Souvatzoglou M, Astner ST, Vaupel P, Nüsslin F, Wilkens JJ, Ziegler SI (2010) Quantitative assessment of hypoxia kinetic models by a cross-study of dynamic ${ }^{18}$ F-FAZA and ${ }^{15} \mathrm{O}-\mathrm{H}_{2} \mathrm{O}$ in patients with head and neck tumors. J Nucl Med 51(9): 1386-1394.

Shibahara I, Kumabe T, Kanamori M, Saito R, Sonoda Y, Watanabe M, Iwata R, Higano S, Takanami K, Takai Y, Tominaga T (2010) Imaging of hypoxic lesions in patients with gliomas by using positron emission tomography with $1-\left(2-\left[{ }^{18} \mathrm{~F}\right]\right.$ fluoro-1-[hydroxymethyl]ethoxy) methyl-2nitroimidazole, a new ${ }^{18} \mathrm{~F}$-labeled 2-nitroimidazole analog: clinical article. J Neurosurg 113(2): 358-368.

Souvatzoglou M, Grosu AL, Ro $\square$ per B, Krause BJ, Beck R, Reischl G, Picchio M, Machulla HJ, Wester HJ, Piert M (2007) Tumour hypoxia imaging with $\left[{ }^{18} \mathrm{~F}\right] \mathrm{FAZA}$ PET in head and neck cancer patients: a pilot study. Eur J Nucl Med Mol Imaging 34(10): 1566-1575.

Swanson KR, Chakraborty G, Wang CH, Rockne R, Harpold HL, Muzi M, Adamsen TC, Krohn KA, Spence AM (2009) Complementary but distinct roles for MRI and ${ }^{18} \mathrm{~F}$-fluoromisonidazole PET in the assessment of human glioblastomas. J Nucl Med 50(1): 36-44.
Takahashi N, Fujibayashi Y, Yonekura Y, Welch MJ, Waki A, Tsuchida T, Sadato N, Sugimoto K, Itoh $\mathrm{H}(2000)$ Evaluation of ${ }^{62} \mathrm{Cu}$ labeled diacetyl-bis(N4-methylthiosemicarbazone) as a hypoxic tissue tracer in patients with lung cancer. Ann Nucl Med 14(5): 323-328.

Tateishi K, Tateishi U, Sato M, Yamanaka S, Kanno H, Murata H, Inoue T, Kawahara N (2013) Application of ${ }^{62} \mathrm{Cu}$-diacetyl-bis (N4- methylthiosemicarbazone) PET imaging to predict highly malignant tumor grades and hypoxia-inducible factor-1a expression in patients with glioma. Am J Neuroradiol 34(1): 92-99.

Thorwarth D, Eschmann SM, Scheiderbauer J, Paulsen F, Alber M (2005) Kinetic analysis of dynamic ${ }^{18} \mathrm{~F}$-fluoromisonidazole PET correlates with radiation treatment outcome in head-and-neck cancer. BMC Cancer 5: 152 .

Thorwarth D, Eschmann SM, Holzner F, Paulsen F, Alber M (2006) Combined uptake of $\left[{ }^{18} \mathrm{~F}\right] \mathrm{FDG}$ and $\left[{ }^{18} \mathrm{~F}\right] \mathrm{FMISO}$ correlates with radiation therapy outcome in head-and-neck cancer patients. Radiother Oncol 80(2): $151-156$.

Thureau S, Chaumet-Riffaud P, Modzelewski R, Fernandez P, Tessonnier L, Vervueren L, Cachin F, Berriolo-Riedinger A, Olivier P, Kolesnikov-Gauthier H, Blagosklonov O, Bridji B, Devillers A, Collombier L, Courbon F, Gremillet E, Houzard C, Caignon JM, Roux J, Aide N, Brenot-Rossi I, Doyeux K, Dubray B, Vera P (2013) Interobserver agreement of qualitative analysis and tumor delineation of ${ }^{18} \mathrm{~F}$ fluoromisonidazole and $3^{\prime}$-deoxy- $3^{\prime}-{ }^{18} \mathrm{~F}$-fluorothymidine PET images in lung cancer. J Nucl Med 54(9): 1543-1550.

Trinkaus ME, Blum R, Rischin D, Callahan J, Bressel M, Segard T, Roselt P, Eu P, Binns D, MacManus MP, Ball D, Hicks RJ (2013) Imaging of hypoxia with ${ }^{18} \mathrm{~F}$-FAZA PET in patients with locally advanced non-small cell lung cancer treated with definitive chemoradiotherapy. J Med Imaging Radiat Oncol 57(4): 475-481.

Valk PE, Mathis CA, Prados MD, Gilbert JC, Budinger TF (1992) Hypoxia in human gliomas: demonstration by PET with fluorine-18fluoromisonidazole. J Nucl Med 33(12): 2133-2137.

Vaupel P, Harrison L (2004) Tumor hypoxia: causative factors, compensatory mechanisms, and cellular response. Oncologist 9(Suppl 5): 4-9.

Vera P, Bohn P, Edet-Sanson A, Salles A, Hapdey S, Gardin I, Ménard JF, Modzelewski R, Thiberville L, Dubray B (2011) Simultaneous positron emission tomography (PET) assessment of metabolism with ${ }^{18}$ F-fluoro-2-deoxy-d-glucose (FDG), proliferation with ${ }^{18} \mathrm{~F}$-fluorothymidine (FLT), and hypoxia with ${ }^{18} \mathrm{~F}$-fluoro-misonidazole (F-miso) before and during radiotherapy in patients with non-small-cell lung cancer (NSCLC): a pilot study. Radiother Oncol 98(1): 109-116.

Vercellino L, Groheux D, Thoury A, Delord M, Schlageter MH, Delpech Y, Barré E, Baruch-Hennequin V, Tylski P, Homyrda L, Walker F, Barranger E, Hindié E (2012) Hypoxia imaging of uterine cervix carcinoma with ${ }^{18}$ F-FETNIM PET/CT. Clin Nucl Med 37(11): 1065-1068.

Wang K, Yorke E, Nehmeh SA, Humm JL, Ling CC (2009) Modeling acute and chronic hypoxia using serial images of ${ }^{18}$ F-FMISO PET. Med Phys 36(10): 4400-4408.

Wilson WR, Hay MP (2011) Targeting hypoxia in cancer therapy. Nat Rev Cancer 11(6): 393-410.

Yamane T, Kikuchi M, Shinohara S, Senda M (2011) Reduction of $\left[{ }^{18} \mathrm{~F}\right]$ fluoromisonidazole uptake after neoadjuvant chemotherapy for head and neck squamous cell carcinoma. Mol Imaging Biol 13(2): 227-231.

Yue J, Yang Y, Cabrera AR, Sun X, Zhao S, Xie P, Zheng J, Ma L, Fu Z, Yu J (2012) Measuring tumor hypoxia with ${ }^{18}$ F-FETNIM PET in esophageal squamous cell carcinoma: a pilot clinical study. Dis Esophagus 25(1): $54-61$.

Zegers CML, Van Elmpt W, Wierts R, Reymen B, Sharifi H, Öllers MC, Hoebers F, Troost EG, Wanders R, van Baardwijk A, Brans B, Eriksson J, Windhorst B, Mottaghy FM, De Ruysscher D, Lambin P (2013) Hypoxia imaging with $\left[{ }^{18} \mathrm{~F}\right] \mathrm{HX} 4$ PET in NSCLC patients: Defining optimal imaging parameters. Radiother Oncol 109(1): 58-64.

Zimny M, Gagel B, DiMartino E, Hamacher K, Coenen HH, Westhofen M, Eble M, Buell U, Reinartz P (2006) FDG - A marker of tumour hypoxia? A comparison with $\left[{ }^{18} \mathrm{~F}\right]$-fluoromisonidazole and $\mathrm{pO}_{2}$-polarography in metastatic head and neck cancer. Eur J Nucl Med Mol Imaging 33(12): 1426-1431. 